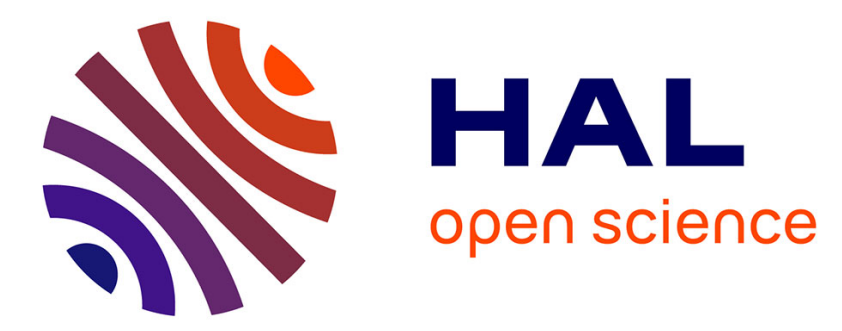

\title{
The time-consistent dial-a-ride problem
}

\section{Oscar Tellez, Samuel Vercraene Vercraene, Fabien Lehuédé, Olivier Péton, Thibaud Monteiro}

\section{To cite this version:}

Oscar Tellez, Samuel Vercraene Vercraene, Fabien Lehuédé, Olivier Péton, Thibaud Monteiro. The time-consistent dial-a-ride problem. Networks, 2022, 79 (4), pp.452-478. 10.1002/net.22063 . hal02460670v2

\section{HAL Id: hal-02460670 \\ https://hal.science/hal-02460670v2}

Submitted on 26 Aug 2021

HAL is a multi-disciplinary open access archive for the deposit and dissemination of scientific research documents, whether they are published or not. The documents may come from teaching and research institutions in France or abroad, or from public or private research centers.
L'archive ouverte pluridisciplinaire HAL, est destinée au dépôt et à la diffusion de documents scientifiques de niveau recherche, publiés ou non, émanant des établissements d'enseignement et de recherche français ou étrangers, des laboratoires publics ou privés. 


\title{
The time consistent dial-a-ride problem
}

\author{
Tellez, Oscar ${ }^{1} \quad$ Vercraene, Samuel $^{1} \quad$ Lehuédé, Fabien ${ }^{2} \quad$ Péton, Olivier $^{2}$ \\ Monteiro, Thibaud ${ }^{1}$ \\ ${ }^{1}$ Laboratoire DISP, INSA Lyon, Villeurbanne, France \\ ${ }^{2}$ IMT Atlantique, LS2N, UMR CNRS 6004, Nantes, France
}

June 2021

\begin{abstract}
In the context of door-to-door transportation of people with disabilities, service quality considerations such as maximum ride time and service time consistency are critical requirements. To identify a good trade-off between these considerations and economic objectives, we define a new variant of the multi-period dial-a-ride problem called the time consistent dial-a-ride problem. A transportation planning is supposed to be time consistent if for each passenger, the same service time is used all along the planning horizon. However, considering the numerous variations in transportation demands over a week, designing consistent plan for all passengers can be too expensive. It is therefore necessary to find a compromise solution between costs and time consistency objectives. The time consistent diala-ride problem is solved using an epsilon-constraint approach to illustrate the trade-off between these two objectives. It computes an approximation of the Pareto front, using a matheuristic framework that combines a large neighbourhood search with the solution of set partitioning problems. This approach is benchmarked on time consistent vehicle routing problem literature instances. Experiments are also conducted in the context of door-to-door transportation for people with disabilities, using real data. These experiments support managerial insights regarding the inter-relatedness of costs and quality of service.
\end{abstract}

Keywords: Vehicle routing, Dial-a-ride problem, Healthcare logistics, Consistency, Set partitioning, Large neighborhood search.

\section{Introduction}

The design of efficient para-transit systems relies both on minimizing operational costs and on providing passengers with an adequate quality of service. In the operations research literature, the Dial-A-Ride Problem (DARP) is a well-known optimization problem that consists in designing minimal-cost vehicle

5 routes to fulfill a set of transportation requests while satisfying a number of service quality requirements. Common applications concern door-to-door transportation of elderly people or people with disabilities. In Medico-Social Institutions (MSI) in France, transportation is considered to be the main expense after wages [1. Transportation plans are defined on a yearly basis and partially revised several times a year whenever necessary. Due to the pressure to cut costs, this is often their main objective, although service quality criteria are also taken into account to define transportation plans.

The DARP formulation considers a single period, typically half a day. Passengers are generally subject to ride-time constraints: they must not be transported longer than a maximum predefined travel duration. In this paper, we examine the case of para-transit systems for people who need to be transported on a regular basis, for example handicapped workers or scholars. The DARP formulation is extended over multiple periods and each period has a known set of transportation requests from passengers.

We carried out a statistical study on field data which shows that $30 \%$ of passengers have a complete and regular schedule throughout the week. The other passengers may have a regular demand only a part of the week, or have slight variations in their planning (change of pickup or destination according to medical appointments, nights spent at the MSI, etc.).

Table 1 shows the percentage of users that have to be transported on five, four,..., one days of the week, respectively. 


\begin{tabular}{lccccc}
\hline Number of demands per week & 5 & 4 & 3 & 2 & 1 \\
\hline Percentage of passengers concerned & $34.51 \%$ & $9.33 \%$ & $11.84 \%$ & $15.42 \%$ & $28.90 \%$ \\
\hline
\end{tabular}

Table 1: Frequency of transportation requests

For medical, cognitive or convenience reasons, it is desirable for passengers with regular demand to be proposed a regular service, i.e. to be picked up at the same time every day in the morning and dropped off at the same time on every evening. Each passenger's demand can have an impact on the schedule of other passengers on the same route. More particularly, the $28.9 \%$ of passengers with one weekly demand do not have consistency requirement, but these passengers "perturbate" the planning of all other passengers and make the consistency issue hard to solve.

During the week, the same fleet of vehicles must satisfy all transportation demands. To approach this objective, it is possible to have different routes and a different number of routes everyday. In the meanwhile, some practical constraints such as passenger time windows or maximum ride times must be respected and the overall transportation cost should be minimized.

This work extends the consistent vehicle routing problem of $[9$ to a consistent dial-a-ride problem. This allows to fully integrate real-life considerations such as time windows at pickup and delivery points, maximum ride times, and routes visiting several delivery points. The objective function to be minimized in [9] is the maximal number of time classes per passenger, where the time classes represents the number of significantly distinct schedules in the week. We reuse this definition of time classes and propose to lexicographically minimize the number of passengers with each number of time classes. In brief, we address a multi-period dial-a-ride problem and study the trade-off between service time consistency and transportation costs. As this problem introduces time consistency within a DARP setting, we call this new variant the time Consistent DARP (TC-DARP).

Section 2 presents how the TC-DARP is related to the existing literature in operations research. In particular, we discuss various definition of time consistency and motivate the use of a lexicographic objective function. In Section 3 , we give a formal definition of the TC-DARP and formulate it as a mixedinteger linear program (MILP) with two objectives: minimizing transportation costs and lexicographically (1) section 4 presents the solution method used to solve the TC-DARP. It a $\varepsilon$-constraint method, called $\operatorname{SP} \varepsilon$ C, that repeatedly solves a set-partitioning (SP) formulation of the TC-DARP. In practice, this set partitioning formulation contains a only a small subset, called pool of routes of all possible routes. This pool of routes is generated by an auxiliary Large neighborhood Search (LNS) algorithm. When the current pool is not enough to ensure a good so time consistency, new routes are appended to this pool. This requires solving an auxiliary optimization problem, which is a DARP with multiple time windows. The generation of these multiple time windows and the adaption of the LNS method used to solve it are presented in Section 5.

In section 6, we present computational results on consistent VRP instances and results on the case study that motivated this work. It concerns the transportation of people with disabilities in the area of

s5 Lyon, France. We collected data from the main carrier in the region 1 , who works for multiple institutions and has a fleet of adapted vehicles. We show how using lexicographic minimization of the number of time classes offers a broader choice to decision makers to achieve a good trade-off between the transportation cost and the quality of service.

\section{Related literature}

60 The research presented in this paper is related to people transportation 6 and more particularly to the DARP, recently surveyed in 13, 23. It also shares some similarities with the School Bus Routing Problem [25] or integrated vehicle scheduling problems arising in bus network design [3, 21], although research in this field seldom focus on modelling each passenger specific constraint. The research papers studying people transportation problems are generally focused on determining the best routes for a set of vehicles in order to serve the transportation requests of a single period. Some consistency aspects are studied in [4] by minimizing the deviation from predefined desired starting time for predefined trips. An alternative model was presented in [28] where the author minimize the deviation from ideal intervals between consecutive service trips of the same link. The consistency aspects in these papers aim at evenly spreading service times at a bus stop over the horizon. Note that we do not allow transfers between

\footnotetext{
${ }^{1}$ Synergihp Rhône-Alpes: www.synergihp-ra.fr
} 
vehicles as it is generally done in public transit systems. The mono-period DARP of our application has been presented in [37. In this paper, we focus on the consistency aspects that appear in the multi-period version of the problem.

The integration of time consistency appeared recently in the vehicle routing problem (VRP) literature. Applications were first identified in the context of fast parcel delivery 11$]$ and were rapidly extended to passenger transportation 9 . Readers interested in an extensive review on vehicle routing with consistency considerations can refer to Kovacs et al. [16. Consistency in vehicle routing problems can be divided into three main categories: service time consistency, driver consistency, and territory consistency. Service time consistency means that regular customers are scheduled to be served at approximately the same time in the planning horizon. As the main focus of our paper, the service time consistency will be detailed in the next section.

Driver consistency consists in minimizing the number of different drivers assigned to each passenger during the planning horizon. The aim is to reinforce the relationship between drivers and passengers in order to improve the quality of service. Braekers and Kovacs [2] computed the average cost of a solution where each passenger was served by one, two and three drivers, respectively, showing that a solution with two drivers can be near optimal whereas solutions with one driver are $10 \%$ costlier on average. Other approaches using soft constraints have yielded similar conclusions [30, 22]. In Feillet et al. [9], drivers are assigned to routes a posteriori, so that service time consistency and driver consistency are considered as independent problems in a lexicographical way.

Territory consistency aims at increasing drivers efficiency through their knowledge of the geographical area in which they operate. A common way of addressing territory consistency is to design independent districts in advance, where independent routing problems are solved every day. This approach was studied in $[18,40,30,29]$.

This paper focuses on service time consistency applied to a Dial-a-Ride Problem (DARP). In contrast to the VRP, the DARP considers one origin and destination for each user and a maximum ride time. The main applications of the DARP concern door-to-door transportation of people, particularly elderly or disabled people [13, 38, 17].

\subsection{Service time consistency models}

Service time consistency consists in serving regular needs at approximately the same hour throughout the whole planning horizon. This is modeled either by hard constraints, that is, imposing an acceptable level of service time variation, or by soft constraints, that is, penalizing service time variations in the objective function.

Groër et al. [11] defined the maximum arrival time variation as the difference between the latest and earliest service times throughout the whole planning horizon, for each customer. This consistent VRP (conVRP) is an extension of the multi-period VRP where the maximal arrival time variation is bounded above by a constant value $L_{\max }$. However, this measure, initially proposed for the small package shipping industry, has some practical drawbacks in the context of passenger transportation.

Feillet et al. [9] define a passenger-oriented time consistency model based on the concept of time classes. They assume that very small variations (e.g. \pm 5 minutes) in service time are not significant for passengers, especially considering approximations and variations due to traffic conditions or unexpected events. Passengers are sensitive to the number of significantly different service times proposed in a week. Similar times are regrouped in the same time class. Consistency is then improved by minimizing the maximum number $C_{\max }$ of time classes over all passengers. The difference between this measure and $L_{\max }$ is highlighted in Figure 1 .

Figures 1(a) and 1(b) represent the service time of a passenger in two distinct solutions. Each vertical line represents the service time from Monday to Friday. In Figure1(a), as service times are evenly spread between 7:00 and 8:00, there are 5 time classes. In Figure 1(b), these times can be grouped into two intervals of 10 minutes: [7:00-7:10] and [7:50-8:00]. This passenger is said to have 2 time classes. While both solutions have the same value $L_{\max }=1$ hour, they do not offer the same consistency to passengers as far as service time is concerned. In our application, a measure based on the number of time classes offers a better quality of service than a solution measure with $L_{\max }$.

There is however one limitation related to objective $C_{\max }$. It is the largest number of time classes in the solution, for all passengers. Hence, a solution with $99 \%$ of passengers who have $C_{\max }$ time classes is equivalent to another solution where only $1 \%$ of passengers are the same situation. In order to overcome this limitation, we propose a lexicographic optimization. We first minimize the number of passengers from the highest to the lowest number of time classes: first passengers with $C_{\max }$ time classes, then 


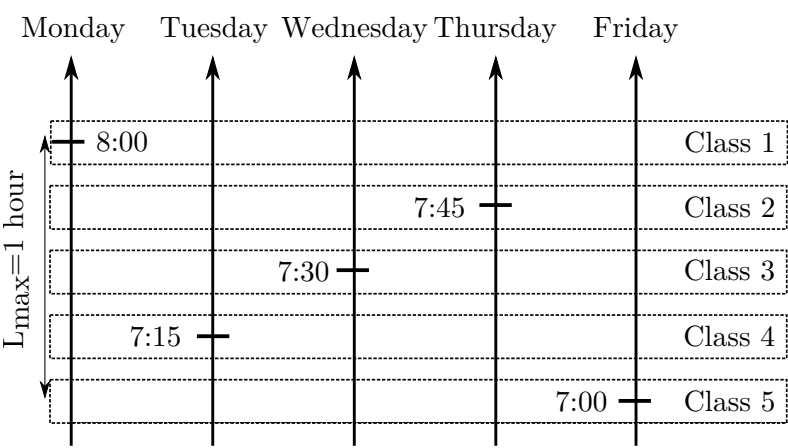

(a) Solution with $C_{\max }=5$ time classes

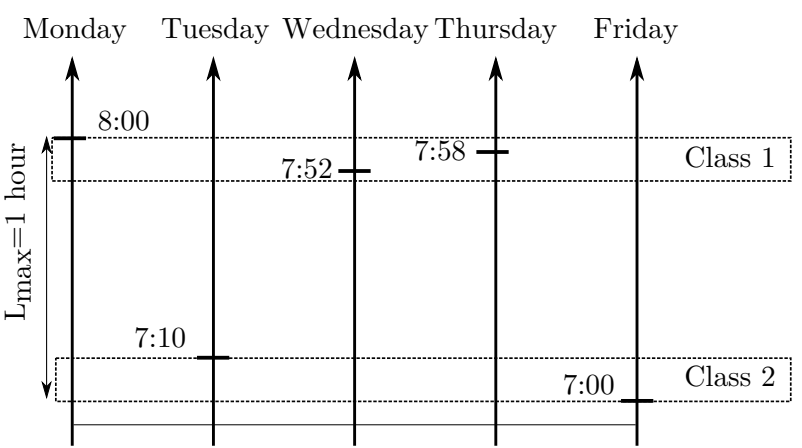

(b) Solution with $C_{\max }=2$ time classes

Figure 1: Comparison, for one passenger, of two solutions having the same $L_{\max }$ but different numbers of time classes. The width of a time class is 10 minutes.

$C_{\max }-1, C_{\max }-2$ and so forth.

To the best of our knowledge, this approach is a new refinement of the Feillet et al. 99 model. Still, according to the passenger transportation company, many good trade-off solutions can be found between the cost optimal solution with $C_{\max }=2$ and the cost optimal solution with $C_{\max }=1$. According to the fair optimization literature [24], the proposed model corresponds to a lexicographic minimax refinement of the min-max model, using counting functions. We show that this lexicographic objective is easily adapted to the context of passenger transportation.

\subsection{Solution approaches for time consistent routing problems}

In the conVRP model introduced by Groër et al. [11], the objective is to optimize service time consistency $\left(L_{\max }\right)$ without compromising a perfect driver consistency (1 driver per customer). These authors proposed a record-to-record travel algorithm and developed benchmark instances for up to 100 customers.

The consistency measure $L_{\max }$ has been used in several subsequent papers (i.e. $34,35,14,15,19$, 39). The current best results on the Groër et al. 11] benchmark instance set was obtained by Xu and Cai 39], who proposed a Variable Neighborhood Search procedure using dedicated local search methods for quickly finding local optima. This approach is based on improving template solutions generated by 3 different shaking methods. A problem extension, denoted the genConVRP, is proposed by Kovacs et al. [15] in which: routes do not necessarily start at the same time, customers are associated with AM/PM time windows, and a maximum number of drivers per customer is defined. Subramanyam and Gounaris [33] propose a branch-and-cut framework to solve the consistent traveling salesman problem which is a randomly generated, with up to 51 customers.

The time Consistent VRP (TCVRP) of Feillet et al. 9 is solved with a dedicated Large Neighborhood Search (LNS) framework. At each iteration, the routes of all periods are destroyed. A VRP with multiple time windows and no waiting time (VRPmTW-nw) is defined in order to decrease the number of time solutions for $C_{\max }=1$ to 5 are saved in the process.

Another related problem is the Time Window Assignment Vehicle Routing Problem (TWAVRP) introduced by Spliet and Gabor 32. In the TWAVRP, a single time window of fixed width has to be assigned to some regular customers before the effective daily demand is known. The assignment is based on a set of demand scenarios, each of which is associated with a given probability. The objective is to minimize the expected transportation cost. The TWAVRP is a particular case of the genConVRP if scenarios are seen as periods and the number of drivers per customer is set to infinity. However, the objective differs: genConVRP optimizes the total transportation cost and TWAVRP the average transportation cost. A branch-price-and-cut algorithm is proposed to optimally solve instances with up to 25 customers. Spliet and Desaulniers [31] propose a variant, called the discrete time window assignment vehicle routing problem, where the chosen time windows are selected from a discrete set. Considering its formulation, the TWAVRP corresponds to the case where each user should have exactly one time class in the TCVRP, with each day in the TCVRP corresponding to a scenario with probability one in the TWARVP.

Consistency issues are also often related to having stochastic customers in the VRP [27]. For example, 
Sungur et al. [34] use a combination of robust optimization in a first phase master problem, and stochastic programming with recourse to daily schedules to address the uncertainty in service times and customer occurrence. Erera et al. [8] investigate the opportunity to give a main fixed route as well as a backup one to frequent customers in a stochastic context.

Finally, the question of service time consistency presents some similarities with some non-periodic applications such as the synchronization of multiple vehicles at the same node. In this case, the arrival time of multiple vehicles at a given location should be synchronized in order to perform a collective operation. The vehicles then continue their routes independently. A survey on synchronization in VRP is given by Drexl [7].

\subsection{Contributions with respect to the literature}

The literature review shows that there is still some gap between what has been proposed in the literature and a practical implementation of time consistency for a DARP application. In this paper, we reuse the notion of time classes introduced in Feillet et al. [9] and extend this previous work from the time consistent VRP to the time consistent DARP. In particular, we consider time windows both at pickup and delivery locations and maximum ride times for all passengers. Another contribution is to better explore solutions where some passengers accept several time classes. To aggregate of the number of time classes over all passengers, we refine the $C_{\max }$ minimization approach of [9]. Our lexicographic approach minimizes the number of passengers with the highest to the lowest number of time classes: first passengers with $C_{\max }$ time classes, then with $C_{\max }-1, C_{\max }-2$ and so forth. As a result, the proposed method finds a more complete Pareto front, showing more trade-off between cost and consistency.

Due to the time windows at delivery locations, routes might contain waiting times. Similarly to Kovacs et al. [15], we consider that the departure time of routes can be changed in order to improve consistency. To achieve these objectives, we revisit the method of [9], in particular by integrating the LNS matheuristic of [37] in the method to design the routes of each week day. As in [9], our algorithm includes a component that recreates the routes of one day, taking account of the times at which users are served on the other days. For this component, a specific case of the DARP with multiple Time Windows is defined. It is solved by adapting the previous LNS algorithm through a new route scheduling algorithm that integrates the constraints and objectives of our problem. The generated routes are combined in an $\varepsilon$-constraint method, with routes selection strategies, to exhibit the TC-DARP Pareto front.

\section{Modeling the time consistent dial-a-ride-problem (TC-DARP)}

In this section, we present the TC-DARP problem settings and give its MILP formulation. Tables 2 , 3 and 4 synthesize the mathematical notations for the sets, data and variables, respectively.

\section{Users}

In the remainder of this paper, we refer to the passengers of the transportation system as users. The set of users is denoted by $\mathcal{U}$. Each user $u \in \mathcal{U}$ is carried between a pickup node $p_{u} \in \mathcal{P}$ and a delivery node $d_{u} \in \mathcal{D}$, where $\mathcal{P}$ and $\mathcal{D}$, denote the set of all pickup and delivery nodes, respectively. Moreover, each user has a maximum ride time $\bar{T}_{u}$, and occupies a particular space $v \in \mathcal{J}$ in vehicles.

Given a planning horizon $\mathcal{T}$ (typically one week), each user can be service at most once in any period, the user's demand is modeled by a vector of binary values of size $|\mathcal{T}|$. More precisely, we define $\beta_{u}^{t} \in\{0,1\}$ for all $u \in U, t \in \mathcal{T}$, where $\beta_{u}^{t}=1$ if user $u$ requires transportation at period $t$ and 0 otherwise. Note that if several users have a common origin or destination, nodes are duplicated so that each pickup node and each delivery node corresponds to exactly one user. Similarly, we only consider users with stable demand within the planning horizon (same pickup, same delivery). Actually, users with several addresses do not expect time consistency. Thus, they are considered as distinct users with separate plannings.

\section{Vehicles}

We assume a homogeneous and unlimited fleet of vehicles based at a single depot $o$. In practice, vehicles are equipped with several spaces (e.g. seats, spaces for wheelchairs) that can receive different categories of users. We thus consider vehicles' capacity as a vector indicating the number of available places in each space. It is denoted by $\mathcal{Q}=\left\{Q_{1}, \ldots, Q_{|\mathcal{J}|}\right\}$, where $\mathcal{J}$ represents the set of passenger spaces (seats, 


\section{Graph representation}

The TC-DARP is defined on a directed graph which node set is $\{\mathcal{P} \cup \mathcal{D} \cup o\}$ and the arcs set containing the following arcs: $(o, i)$ where $i \in \mathcal{P} ;(i, j)$ where $i, j \in \mathcal{P} \cup \mathcal{D}, i \neq j$; and $(i, o)$ where $i \in \mathcal{D}$. Each node $i$ is associated with a service duration $\zeta_{i}$ and a time window $\left[a_{i}, b_{i}\right]$. Every arc $(i, j)$ represents the fastest path from node $i$ to node $j$ and is associated with a travel time $t_{i j}$ and a distance $d_{i j}$.

\section{Routes}

We propose a route-based MILP formulation of the TC-DARP. The set of all routes is denoted by $\Omega$. A route $\omega \in \Omega$ is any feasible sequence of nodes visited by the same vehicle. More precisely, a route is feasible if it starts and finishes at the depot $o$, serves a set of users by visiting their pickup node and their delivery node (in this order), while respecting time windows at all nodes visited, users ride time constraints and vehicle capacity constraints. Given a potentially large set of feasible routes, the MILP model aims at selecting a subset of routes that cover the whole demand. The binary decision variable $y_{\omega}^{t}$ is equal to 1 if route $\omega \in \Omega$ is selected at period $t \in \mathcal{T}$.

Each selected route is associated with one vehicle. For each route, we consider three types of costs: a fixed cost $\lambda$ paid if the route is used at least once in the planning horizon, a cost per kilometer $\tau$ related the fuel consumption, and a cost per hour $\alpha$ related to the driver's cost. For a given route $\omega$ which length and duration can be easily calculated, the sum of fuel and driver's related cost is denoted by $\pi_{\omega}$.

\section{Routes scheduling}

The pickup and delivery nodes $i \in \mathcal{P} \cup \mathcal{D}$ on a route $\omega$ are associated with a time $H_{i, \omega} . H_{i, \omega}$ is the earliest possible service time of node $i$ in a schedule of $\omega$ that minimizes its duration, satisfies time windows at each node and users maximum ride time constraints. In order to improve time consistency, the departure of a route can be postponed provided the route remains feasible. The maximal possible shift for a route $\omega \in \Omega$ is denoted by $\Delta_{\omega}^{+}$. The shift of route $\omega \in \Omega$ departure time at period $t \in \mathcal{T}$ is expressed by continuous variables $\delta_{\omega}^{t} \in\left[0, \Delta_{\omega}^{+}\right]$. Adding waiting time to improve consistency is possible only at the depot. More precisely, once vehicles have left the depot, waiting times are introduced only when the vehicle arrives at some pickup or delivery point before the opening of time windows. Other waiting times are not authorized, even though they would sometimes improve consistency. This rule is realistic with respect to drivers practice. Indeed, a driver would not wait at a pickup location within the user's time windows, possibly with other users on board, just to improve its consistency. Appendix A.2 details the

245 scheduling procedure for the calculation of $\Delta_{\omega}^{+}$and the satisfaction of the time windows and ride-time constraints.

\section{Time classes}

The set of selected routes determines the service times for all users. Given these service times, it is possible to assign each visit to a particular user to time classes. The binary variable $z_{u c}^{t}$ is equal to 1 if user $u \in \mathcal{U}$ is assigned to time class $c \in \mathcal{C}$ at period $t \in \mathcal{T}$, and 0 otherwise. For example, in Figure 1.b), Tuesdays and Fridays belong to class 1. Thus, assuming that the corresponding user is denoted $\overrightarrow{\bar{u}}$, we have $z_{\bar{u} 1}^{2}=z_{\bar{u} 1}^{5}=1$. Similarly, Mondays, Wednesdays and Thursdays belong to class 2 , then $z_{\bar{u} 2}^{1}=z_{\bar{u} 2}^{3}=z_{\bar{u} 2}^{4}=1$.

Binary variables $\mu_{u c}$ indicate which time classes from set $\mathcal{C}$ are actually used by user $u$. In the preceding example, the user $\bar{u}$ has two classes, thus $\mu_{\bar{u} 1}=\mu_{\bar{u} 2}=1$. Without loss of generality, we assume that if a user has $\bar{c}$ time classes $(1 \leq \bar{c} \leq 5)$, these time classes are sequentially numbered from 1 to $\bar{c}$.

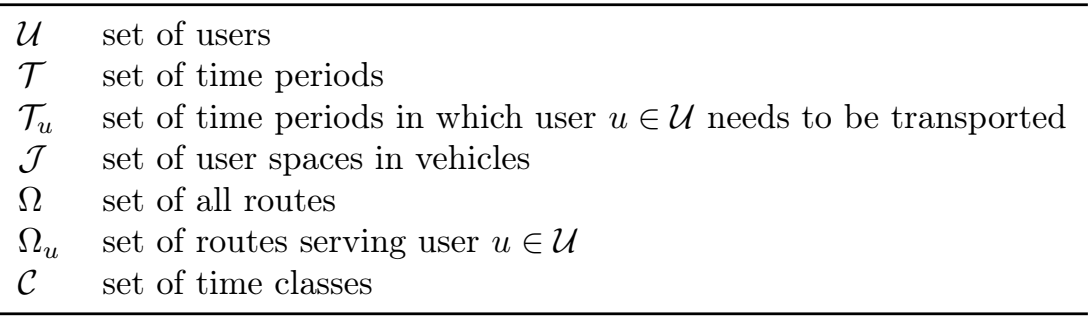

Table 2: Sets 


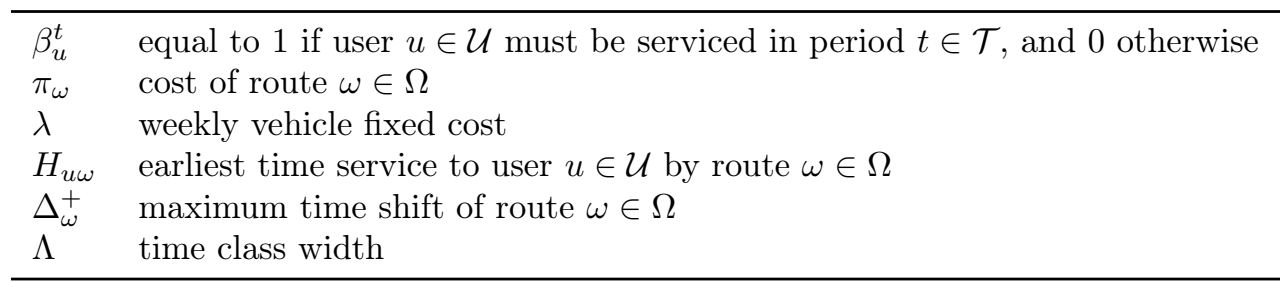

Table 3: Data

\begin{tabular}{ll}
\hline Binary Variables \\
\hline$y_{\omega}^{t} \in\{0,1\} \quad=1$ if route $\omega \in \Omega$ is selected at period $t \in \mathcal{T}$, and 0 otherwise \\
$z_{u c}^{t} \in\{0,1\} \quad=1$ if user $u \in \mathcal{U}$ is assigned to time class $c \in \mathcal{C}$ at period $t \in \mathcal{T}$, and 0 otherwise \\
$\mu_{u c} \in\{0,1\} \quad=1$ if user $u \in \mathcal{U}$ uses time class $c \in \mathcal{C}$, and 0 otherwise \\
\hline \multicolumn{1}{c}{ Other variables } \\
\hline$\delta_{\omega}^{t} \in\left[0, \Delta_{\omega}^{+}\right]$ & time shift (used margin) of route $\omega \in \Omega$ at period $t \in \mathcal{T}$ \\
$s_{u c}^{-}, s_{u c}^{+} \in \mathbb{R}^{+}$ & lower and upper bounds for the time class $c \in \mathcal{C}_{u}$ for user $u \in \mathcal{U}$ \\
$h_{u}^{t} \in \mathbb{R}^{+}$ & beginning of service for user $u \in \mathcal{U}$ at period $t \in \mathcal{T}$ \\
$\nu$ & number of vehicles needed for the whole planning horizon \\
$m_{c}$ & number of users having $c \in \mathcal{C}$ time classes (post-processed variable) \\
\hline
\end{tabular}

Table 4: Summary of variables

\section{Objective functions}

The TC-DARP is a bi-objective problem which consists in selecting a subset of routes from $\Omega$ such that transportation requests on the planning horizon are satisfied within their time windows and maximum ide times.

The first objective is the minimization of transportation costs, that include the fleet fixed cost $\lambda \nu$ and the cost of all routes used in the solution.

$$
\min f=\lambda \nu+\sum_{\omega \in \Omega} \sum_{t \in \mathcal{T}} \pi_{\omega} y_{\omega}^{t}
$$

The second objective minimizes time inconsistency, which is modeled with a lexicographical refinement of the time class model proposed by Feillet et al. [9]. The expression used in the MILP model is the following:

$$
\operatorname{lexmin} \hat{\boldsymbol{g}}=\left(\sum_{u \in \mathcal{U}} \mu_{u|\mathcal{C}|}, \ldots, \sum_{u \in \mathcal{U}} \mu_{u 2}\right) .
$$

This expression lexicographically minimizes the number of people having more than $c$ time classes, where $c$ decreases from $|\mathcal{C}|$ to 2 . The expression $\sum_{u \in \mathcal{U}} \mu_{u c}$ counts the number of users with $c$ or more time classes. This is equivalent to the lexicographical minimization of the number of users whose number of time classes is exactly $|\mathcal{C}|,|\mathcal{C}|-1$, down to 1 , respectively. In the remainder of the paper, we denote by

$$
\operatorname{lexmin} \boldsymbol{g}=\left(m_{|\mathcal{C}|}, \ldots, m_{1}\right)
$$

the alternative formulation of this objective, where $m_{c}$ denotes the number of users having $c$ time classes. It is post-processed from the values of the $\mu_{u c}$ variables using the following expressions:

$$
\left\{\begin{array}{l}
m_{1}=|\mathcal{U}|-\sum_{u \in \mathcal{U}} \mu_{u 2} \\
m_{c}=\sum_{u \in \mathcal{U}} \mu_{u c}-\sum_{u \in \mathcal{U}} \mu_{u, c+1} \quad \forall c \in\{1, \ldots,|\mathcal{C}|-1\} \\
m_{|\mathcal{C}|}=\sum_{u \in \mathcal{U}} \mu_{u|\mathcal{C}|}
\end{array}\right.
$$

\section{Constraints}

The set of TC-DARP feasible solutions is defined by the following constraints: 


$$
\begin{gathered}
\sum_{\omega \in \Omega_{u}} y_{\omega}^{t}=\beta_{u}^{t} \quad \forall u \in \mathcal{U}, t \in \mathcal{T}, \\
\sum_{\omega \in \Omega} y_{\omega}^{t} \leq \nu \quad \forall t \in \mathcal{T}, \\
\sum_{c \in \mathcal{C}} z_{u c}^{t}=1 \quad \forall u \in \mathcal{U}, t \in \mathcal{T}_{u}, \\
h_{u}^{t}=\sum_{\omega \in \Omega_{u}}\left(H_{u \omega} y_{\omega}^{t}+\delta_{\omega}^{t}\right) \quad \forall u \in \mathcal{U}, t \in \mathcal{T}_{u}, \\
\delta_{\omega}^{t} \leq \Delta_{\omega}^{+} y_{\omega}^{t} \quad \forall \omega \in \Omega, t \in \mathcal{T}, \\
s_{u c}^{-} \leq h_{u}^{t}+M\left(1-z_{u c}^{t}\right) \quad \forall c \in \mathcal{C}, u \in \mathcal{U}, t \in \mathcal{T}_{u}, \\
h_{u}^{t} \leq s_{u c}^{+}+M\left(1-z_{u c}^{t}\right) \quad \forall c \in \mathcal{C}, u \in \mathcal{U}, t \in \mathcal{T}_{u}, \\
s_{u c}^{+}-s_{u c}^{-}=\Lambda \quad \forall c \in \mathcal{C}, u \in \mathcal{U}, \\
s_{u c}^{+} \leq s_{u, c+1}^{-} \quad \forall c \in \mathcal{C} /\{|\mathcal{C}|\}, u \in \mathcal{U}, \\
\sum_{\omega \in \Omega_{u}} y_{\omega}^{t}=\sum_{c \in \mathcal{C}} z_{u c}^{t} \quad \forall u \in \mathcal{U}, t \in \mathcal{T} \mathcal{T}_{u}, \\
z_{u c}^{t} \leq \mu_{u c} \quad \forall c \in \mathcal{C}, u \in \mathcal{U}, t \in \mathcal{T}_{u}, \\
\mu_{u, c+1} \leq \mu_{u c} \quad \forall c \in \mathcal{C}, \forall u \in \mathcal{U}, \forall t \in \mathcal{T}, \\
y_{\omega}^{t}, z_{u c}^{t}, \mu_{u c} \in\{0,1\} \quad \forall c \in \mathcal{C}, u \in \mathcal{U}, t \in \mathcal{T}, \omega \in \Omega, \\
\delta_{\omega}^{t}, h_{u}^{t} s_{u c}^{-}, s_{u c}^{+}, \nu \in \mathbb{R}^{+} \quad \forall c \in \mathcal{C}, u \in \mathcal{U}, t \in \mathcal{T}, \omega \in \Omega .
\end{gathered}
$$

Constraints (5) are partitioning constraints ensuring the satisfaction of the users demand. Constraints (6) count the number of vehicles needed during the planning horizon. Constraints (7) state that each user served in period $t \in \mathcal{T}$ should be given a single time class. Constraints (8) determine the service time for each user of route $\omega$ when its departure is shifted by the value $\delta_{\omega}^{t}$. Constraints (9) state that if a route $\omega$ is selected in period $t$, then its time shift $\delta_{\omega}^{t}$ should not exceed the value $\Delta_{\omega}^{+}$.

Constraints (10) and (11) linearize the following logical expression:

$$
z_{c u}^{t}=1 \Rightarrow s_{u c}^{-} \leq h_{u}^{t} \leq s_{u c}^{+} \quad \forall u \in \mathcal{U}, t \in \mathcal{T}_{u}, c \in \mathcal{C} .
$$

They state that if a user $u$ is assigned to the time class $c$ at period $t$, then its service time should be within the bounds of this time class $c$. Constraints 112 set the width of a time class. Constraints 13 . avoid overlap between time classes. Constraints (14) link the number of routes that serve one given user and the number of time class variables. Constraints (15) define variables $\mu_{u c}$ necessary for counting the number of time classes of each user. Constraints (16) ensure that time classes are defined in increasing order. For example, time class \#2 is allocated to a user only if time class \#1 already exists and is not compatible with a given service time. Finally, the definition of variables is given by constraints (17) and (18).

\section{Solution method}

This section presents the Set Partitioning-based $\varepsilon$-constraint matheuristic, denoted $\mathrm{SP} \varepsilon \mathrm{C}$, that has been designed to solve the bi-objective TC-DARP. This method iteratively solves Set Partitioning Problems (SPPs) in an $\varepsilon$-constraint framework. SPPs correspond to a route-based formulation of the TC-DARP considering a subset of the whole set of feasible routes. This subset of routes, called pool of routes, contains routes generated by an auxiliary heuristic solution method to solve the TC-DARP. Here we generate the pool of routes by using a Large Neighborhood Search algorithm (LNS). This section is structured as follows: Section 4.1 presents the general framework of $\mathrm{SP} \varepsilon \mathrm{C}$, that traces a Pareto front approximation between the two objectives of the TC-DARP. Section 4.2 details the optimization procedure for solving the TC-DARP with a single objective. Next subsections are focus on two more specific topics: the initialization of the SP $\varepsilon$ Cframework (Section 4.3), the selection of the pool of routes from a larger set of routes (Section 4.4). 


\subsection{The $\operatorname{SP} \varepsilon \mathrm{C}$ matheuristic framework}

The general framework, introduced in Algorithm 1, is based on an $\varepsilon$-constraint procedure [12, 5]. It finds an approximation of the Pareto front between the two objectives of the TC-DARP: the transportation cost $f$, and the time inconsistency $\boldsymbol{g}$. This is illustrated in Figure 2 .

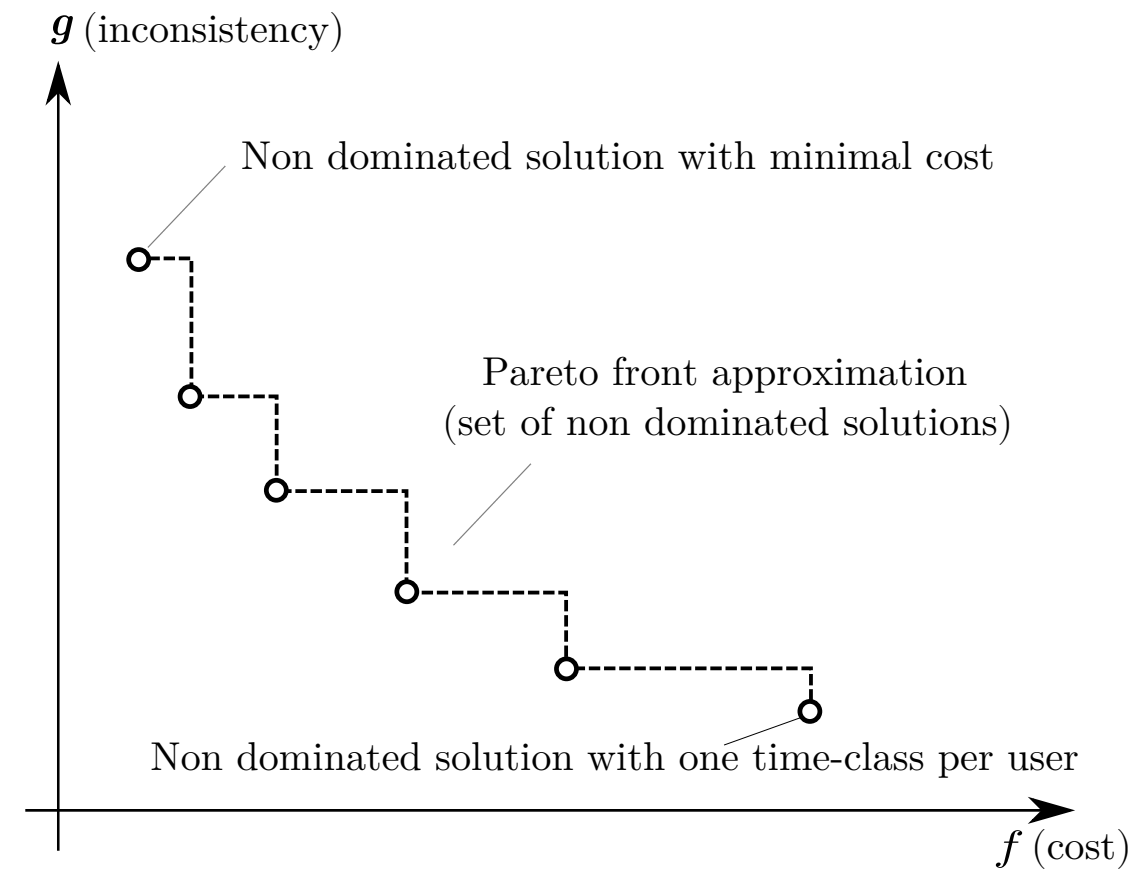

Figure 2: Pareto front approximation designed by the $\mathrm{SP} \varepsilon \mathrm{C}$ algorithm solutions found so far that contains these routes, and the first $N_{\max }$ routes are kept (line 16.

Since the fleet size is not limited, there is an extreme point in the Pareto front such that every user has only one time class (i.e. $\boldsymbol{g}=(0, \ldots,|\mathcal{U}|))$. Therefore the stopping criterion used in line 6 is met when

In a nutshell, the algorithm starts from the best solution found by lexicographically minimizing cost and then inconsistency. Then, the inconsistency objective is progressively improved by allowing an increase of the transportation cost by $\varepsilon$ percent. Every time a new non-dominated solution is found, it is stored in the Pareto front approximation. The procedure stops when every user has 1 time class.

Algorithm 1 presents the $\mathrm{SP} \varepsilon \mathrm{C}$ framework, the $\varepsilon$-constraint matheuristic. In this algorithm, a pool of routes $\mathcal{L}$ is generated together with the initial solution. Two types of solution are used, a temporary solution $S$ (line 3) and a best found solution $S^{*}$. They are initialized with the procedure described in Section 4.3 (line 4). This procedure solves a multi-period DARP in which cost $f$ is minimized. The routes found while solving this multi-period DARP are appended to the pool $\mathcal{L}$. The cost of solution $S^{*}$ is taken as the cost upper limit $\bar{f}$ (line 5 ).

Lines 7 to 15 describe an iteration of the algorithm. The procedures described in lines 7 and 8 aim at finding a new temporary solution $S$, as detailed in Figure 3 . First inconsistency $\boldsymbol{g}$ is minimized subject to a maximal cost constraint. Given that this procedure starts with a feasible solution $S^{*}$, it results in a solution $S$ such that $\boldsymbol{g}(S) \leq_{l e x} \boldsymbol{g}\left(S^{*}\right)$. Then the cost objective $f$ is minimized subject to a maximal inconsistency level $\boldsymbol{g}(S)$. During these two procedures, pool $\mathcal{L}$ is updated with new routes.

If the temporary solution $S$ is strictly better than $S^{*}$ for at least one of the objectives (i.e. if $f(S)<f\left(S^{*}\right)$ or $\boldsymbol{g}(S)<_{\text {lex }} \boldsymbol{g}\left(S^{*}\right)$, line 9 then solution $S^{*}$ is updated with $S$ (line 10) and $S^{*}$ is added to the Pareto front approximation (line 11). Otherwise, the value of $\varepsilon$ is geometrically increased by a factor $\phi$ (line 13). At the end of an iteration, the cost limit $\bar{f}$ is updated based on the cost of $S^{*}$ (line 15) and $\varepsilon$ value.

Note that the size of pool $\mathcal{L}$ increases at each iteration, which can eventually cause memory issues. Thus, the routes in $\mathcal{L}$ are ordered in non-decreasing order of the consistency measure among all TC-DARP $\boldsymbol{g}=(0, \ldots,|\mathcal{U}|)$ 

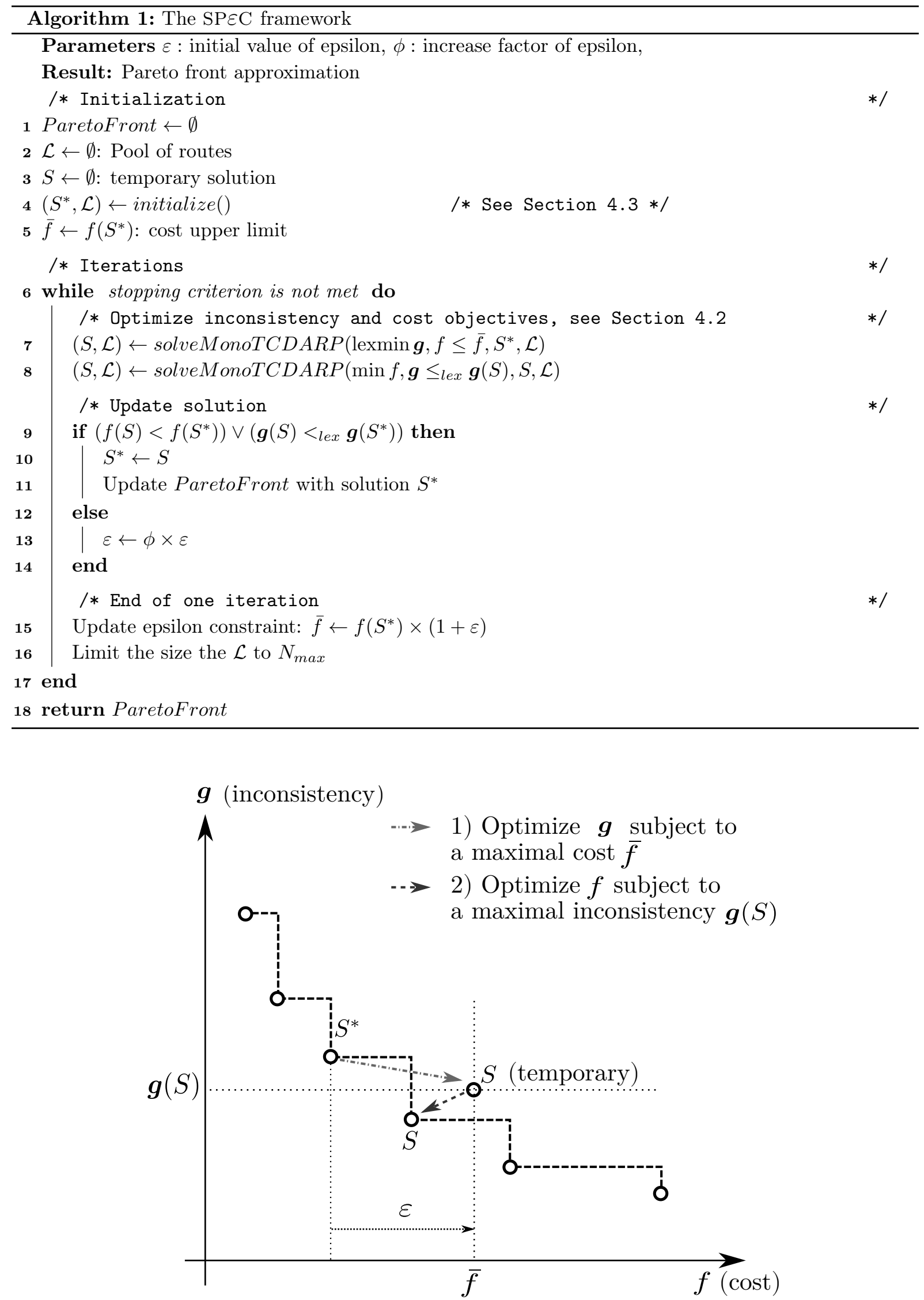

Figure 3: Pareto front exploration with the two optimization procedures of one iteration (Algorithm 1 lines 7 and 8 . 


\subsection{Mono-objective optimization procedure}

Line 7 of Algorithm 1 concerns the minimization of inconsistency subject to a maximal cost constraint. Line 8 concerns the minimization of cost subject to a maximal inconsistency constraint. These two consistent DARP formulations with a single objective are solved with Algorithm 2 The arguments of Algorithm 2 are the objective function $z$ to be minimized ( $z$ is a generic notation representing either $f$ or $\boldsymbol{g}$ ), an upper bound $\mathcal{E}$ on the other objective, a solution $S_{0}$ and a pool of routes $\mathcal{L}$.

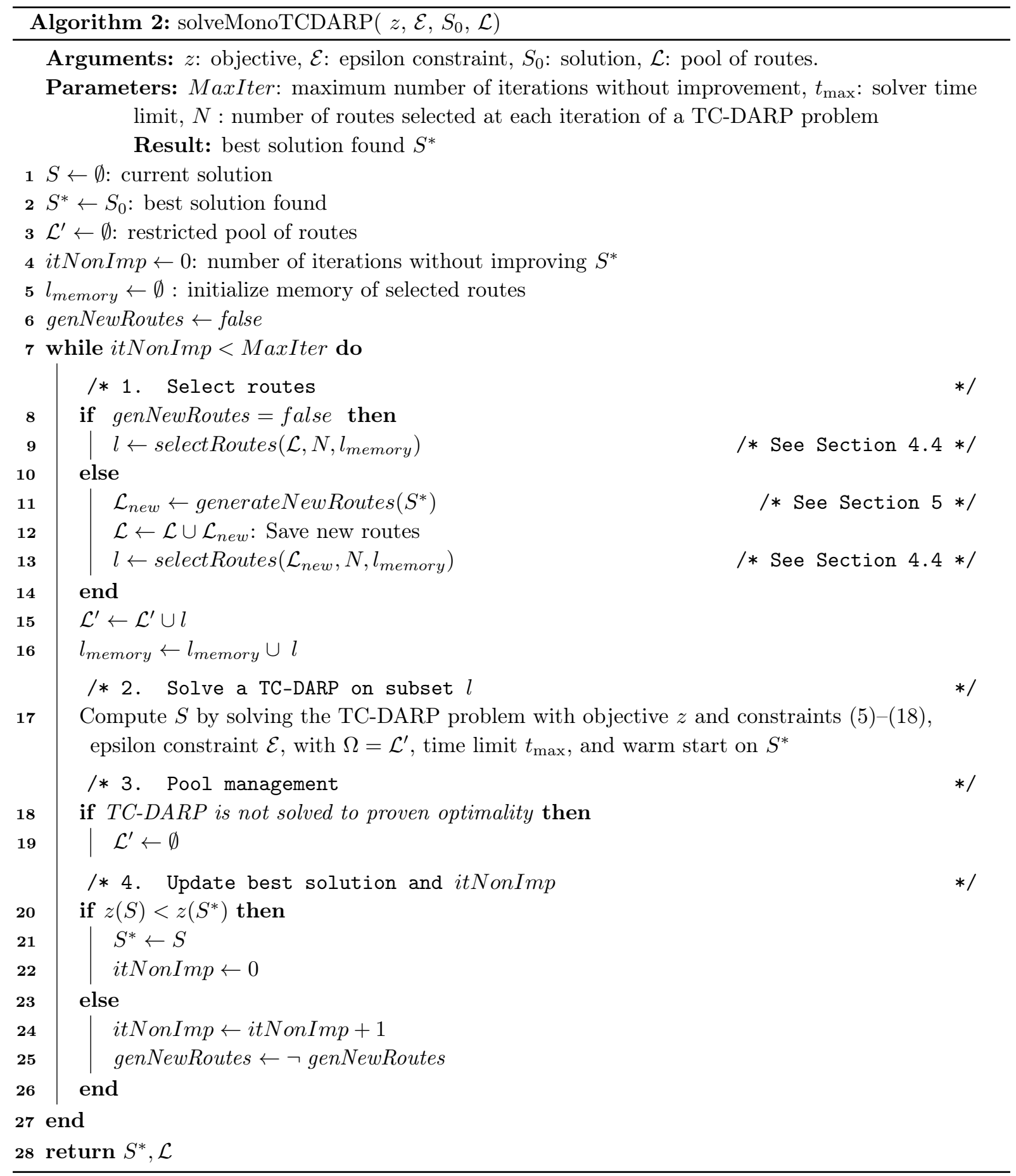

In lines 1 to 5 , the main variables are initialized. The current solution $S$, a restricted pool of routes $\mathcal{L}^{\prime} \subset \mathcal{L}$ and a list of selected routes are initialized as empty sets. The best solution found $S^{*}$ is initialized to $S_{0}$. The variable itNonImp, initialized with the value 0 , counts the number of iterations with no improvement of the objective function. Finally, the boolean variable genNewRoutes indicates if the pool $\mathcal{L}$ must be enriched with new routes. It is initialized with the value FALSE. 
Each iteration of Algorithm 2 consists of four steps: 1) selecting a subset of routes from $\mathcal{L} ; 2$ ) solving the mono-objective TC-DARP given the set of selected routes; 3) managing the pool of routes; and 4) updating the best solution. This process iterates until MaxIter iterations without any improvement of $S^{*}$ having been performed.

In Step 1, a subset $l$ of $N$ routes is selected from a larger pool of routes, which is either $\mathcal{L}$ (line 9 ) or a new pool $\mathcal{L}_{\text {new }}$ determined from the set of service times in solution $S^{*}$ (lines 11 13). The process followed to generate these new routes is detailed in Section 5 . New routes in $\mathcal{L}_{\text {new }}$ are saved in $\mathcal{L}$ (line 12 to enable their selection in further iterations. The procedure for selecting the $N$ routes is the same regardless of the pool. It is called selectRoutes and it is detailed in Section 4.3. In this procedure, routes are selected based on their performance and whether they were chosen on a previous iteration. Selected routes are then saved in memory at every iteration (line 16 ).

Step 2 consists in solving a TC-DARP defined by the objective function $z$ and constraints $(5$-18), with epsilon constraint $\mathcal{E}$, where the set of routes $\Omega$ is the restricted pool $\mathcal{L}^{\prime}$. This model is solved with

345 a time limit set at $t_{\max }$ and a warm start on $S^{*}$. Depending whether $z$ represents $f$ or $\boldsymbol{g}$, the objective $z$ to be minimized is either the cost or the inconsistency. In Step 3 (lines 18 19), the restricted pool of routes is cleared if the TC-DARP at Step 2 could not be solved to proven optimality. This mechanism, inspired by previous research [10,37, keeps the pool size manageable.

Step 4 updates the current best solution $S^{*}$ and the counter itNonImp of iterations without improvement. Thanks to the warm start, the value of the objective function $z(S)$ cannot increase from one iteration to another. Given this property, when the value of $z\left(S^{*}\right)$ has not been improved for MaxIter iterations, we suppose that the algorithm has reached a local optimum. The parameter itNonImp is used as a stopping criterion. When the solution is not improved, the value of the boolean genNewRoutes is modified in order to diversify the process by switching between pools $\mathcal{L}$ and $\mathcal{L}_{\text {new }}$ in step 1.

\subsection{Initialization}

The initial solution of Algorithm 1 can be obtained by executing a variant of Algorithm 2 ignoring all consistency considerations. This amounts to solve a separate dial-a-ride problem for each time period and the corresponding mathematical model, called MP-DARP (multi-period DARP) is as follows:

$$
\begin{array}{lr}
\min f=\lambda \nu+\sum_{\omega \in \Omega} \sum_{t \in \mathcal{T}} \pi_{\omega} y_{\omega}^{t} & \\
\text { s.t. } & \forall u \in \mathcal{U}, t \in \mathcal{T}_{u} \\
\sum_{\omega \in \Omega_{u}} y_{\omega}^{t} \geq 1 & \forall t \in \mathcal{T} \\
\sum_{\omega \in \Omega} y_{\omega}^{t} \leq \nu & \forall \omega \in \Omega, t \in \mathcal{T} \\
y_{\omega}^{t} \in\{0,1\} & \\
\nu \in \mathbb{N} & \forall
\end{array}
$$

The objective function (20) is the same as Equation (1). Constraints (21) are set covering constraints for demand satisfaction. Constraints $(22)$ enforce the number of vehicles to be less than or equal to $\nu$ at each period.

The MP-DARP model is separable with respect to the variables $y_{\omega}^{t}$. This set covering formulation is equivalent to $|\mathcal{T}|$ independent DARP route based formulations.

It can be solved to optimality by a solver provided the number of routes $|\Omega|$ remains reasonable. Rather than directly using a solver, we first run the Large Neighborhood Search (LNS) method proposed by Tellez et al. 37] in order to quickly generate good solutions with feasible routes of minimal duration. For the sake of completeness, we summarize the content of this previous contribution: The LNS algorithm iteratively destroys and repairs parts of the current solution using several operators. It is hybridized with a set partitioning component. This component solves a set partitioning problem to reassemble routes 370 that were generated at distinct iterations of the LNS. A reactive mechanism automatically adjusts its parameters. On the LNS side, the classical "best insertion" and "k-regret" operators are used to repair solutions. To destroy a solution, we found that, in combination with the set partitioning component, only those two destroy operators were needed. These are the random removal and history node-pair removal operators. The paper investigates the use of reconfigurable vehicles, which facilitates the transportation of passengers with wheelchairs. An efficient feasibility checking algorithm is proposed to handle this 
feature in repair operators. The route scheduling algorithm minimizes the route duration and checks time windows and maximal ride time constraints. The method was evaluated on real-life instances as well as academic instances of the DARP and was shown to be competitive with other state-of-the-art metaheuristics.

We use the LNS algorithm as a black box that is able to generate a number of good solutions to the DARP. These solutions contains routes that we store in a set $\Omega^{\prime} \subset \Omega$. The MP-DARP is then solved with $\Omega^{\prime}$ instead of the untractable set $\Omega$. Each time $\Omega^{\prime}$ is updated, dominated routes (i.e. routes $\omega \in \Omega^{\prime}$ which users are included in another route $\omega^{\prime} \in \Omega^{\prime}$ and which cost is greater than $\omega^{\prime}$ ) are filtered out.

Given that the MP-DARP is a set covering formulation, a solution $S$ may contain users' demands served by more than one route. In this case, removing one of the duplicated visits reduces the solution cost (the triangular inequality is assumed).

A solution $S$ is repaired by updating $\Omega^{\prime}$ as follows. For each user $u$ visited more than once in $S$ and for each route $\omega \in \Omega^{\prime}$ that visits user $u$, a new route $\omega^{\prime}$ identical to route $\omega$ but that does not visit user $u$ is added to $\Omega^{\prime}$. Then, the MP-DARP formulation is solved again on the updated set of routes $\Omega^{\prime}$ and a warm-start on $S$.

\subsection{Selection of routes: function $\operatorname{selectRoutes}\left(\mathcal{L}, N, l_{\text {memory }}\right)$}

When the current pool of routes $\mathcal{L}$ or $\mathcal{L}_{\text {new }}$ is too large, the MILP solver cannot improve the given initial solution. Hence, it becomes necessary to select a subset of routes of reasonable size. The function selectRoutes, called at lines 9 and 13 of Algorithm 2 is used to randomly select $N$ from a larger set $\mathcal{L}$. This process uses the set $l_{\text {memory }}$ of the routes previously selected at former calls of this function.

First, all routes in $\mathcal{L}$ are given a score. When the objective function is $f$, this score of a route corresponds to the cost of the best TC-DARP solution found so far that uses this route in at least one period. When the objective function is $\boldsymbol{g}$, the score of a route is the consistency measure among all TC-DARP solutions found so far that use this route in at least one period. Then, $\mathcal{L}$ is sorted according to these scores in non-decreasing order.

The route selection process browses the sorted pool of routes. For each route, we check whether it was already selected in a previous call. In this case, it is selected again with a probability $\gamma$. Otherwise, it is systematically selected. The process stops when $N$ routes have been selected.

Figure 4 shows an example of the route selection with $N=6$. Each line represents the sorted pool of routes. Each square represents a route in this sorted pool. The first call selects the first six routes. In the second call, the grey area represents routes that have been selected at the first call. The second call randomly selects 1 route in the grey area and completes the selection with the first five routes in the white area. The third call randomly selects two routes in the grey area and completes the selection with the first four routes in the white area.

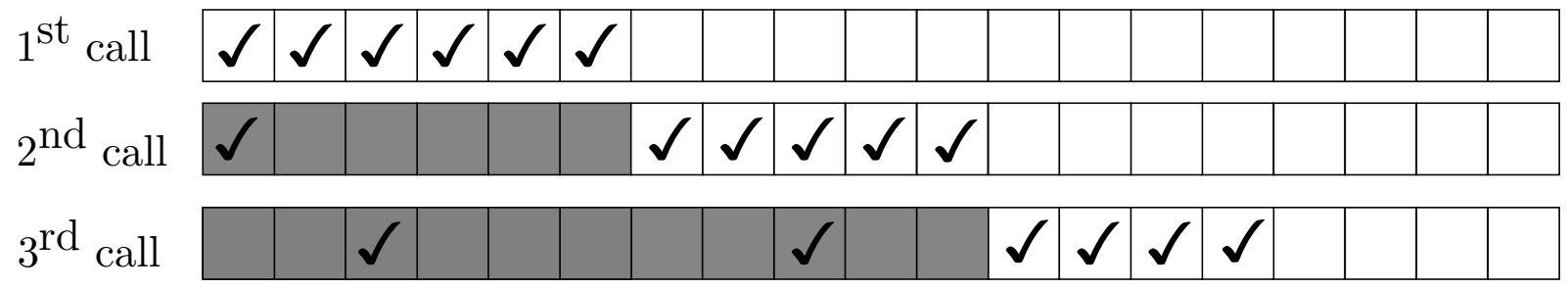

$(\ldots)$

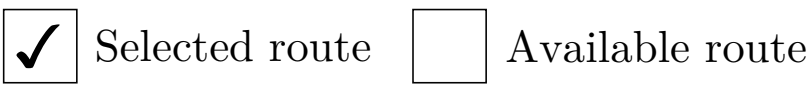

Route selected in previous calls

Figure 4: Example of 3 successive calls to the sequential route selection with $N=6$.

The sequential route selection process increases the probability of selecting together routes that have been part of the same solution with the same overall cost-based or consistency-based performance. The routes must be sorted each time the sequential route selection is called, as performance indicators may change from one iteration to another.

The practical implementation of the selectRoute function includes an extension of the $N$ selected routes: the set of selected routes is augmented by projection and complementary routes, defined by Definition 4.1 
Definition 4.1. Projection and complementarity A route $\omega^{\prime}$ is called a projection of a route $\omega$ in period $t \in \mathcal{T}$ if it contains only the users of $\omega$ who have a demand in period $t$, in the same sequence as in $\omega$. The route $\omega^{\prime \prime}=\omega \backslash \omega^{\prime}$ is called the complementary route of $\omega^{\prime}$.

The definition 4.1 is illustrated by Figure 5 . At period $t$, Route $\omega$ starts from depot D, serves user requests 1,2 and 3 and returns to the depot. If users 1 and 2 have a transportation request at period $t^{\prime}$ and user 3 does not, the route $\omega^{\prime}$ serving user requests 1 and 2 is the projection of $\omega$ and the route $\omega^{\prime \prime}$ serving request 3 is the complementary of $\omega^{\prime}$.

route for period $t$

projection route for period $\mathrm{t}^{\prime}$

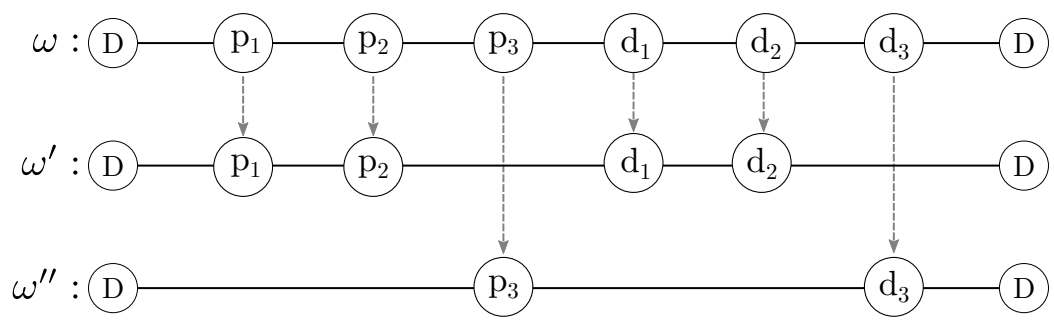

Figure 5: Example of projection and complementary routes

\section{Generation of new routes: the DARP with multiple time win- dows}

In section 4.3 it is explained how the $\mathrm{SP} \varepsilon \mathrm{C}$ framework is initialized with routes that are generated by solving independent DARP instances with an LNS algorithm. These routes are combined in order to form more consistent solutions. In Algorithm 2 (line 11), the time consistency can nevertheless be improved by generating new routes, denoted $\mathcal{L}_{\text {new }}$, that are not likely to be generated by the LNS operators.

This section introduces a new optimization problem: the dial-a-ride problem with multiple time windows (DARPmTW). To the best of our knowledge, this problem has never been treated in the literature. In order to better explain how the the DARPmTW arises from the TC-DARP, we first give an example of multiple time windows for one particular user in Section 5.1. In section 5.2 we explain how times windows are generated for all users and how these time windows are embedded in DARPmTW instances. Section 5.3 focuses on the solution methods to solve the DARPmTW.

\subsection{Multiple time windows for a given user}

Let us consider a user $u \in \mathcal{U}$ that must be visited in the time window $\left[a_{p_{u}}, b_{p_{u}}\right]$. In the current solution, the variables $h_{u}^{t}$ represent the service time of user $u$ at time period $t \in \mathcal{T}_{u}$. The set of service times of user $u$ is denoted $\mathcal{H}_{u}$ and its number of time classes is denoted by $\mu\left(\mathcal{H}_{u}\right)$.

Figure 6 represents the user's schedule. The horizontal axis represents the time window $\left[a_{p_{u}}, b_{p_{u}}\right]$ and the five service times that can be regrouped in 3 time classes of width $\Lambda$ : class 1 includes $h_{u}^{1}, h_{u}^{2}$ and $h_{u}^{3}$ while isolated values $h_{t}^{4}$ and $h_{t}^{5}$ define two time classes on their own. Note that the service times are regrouped in time classes with the same greedy algorithm as in Feillet et al. 9 .

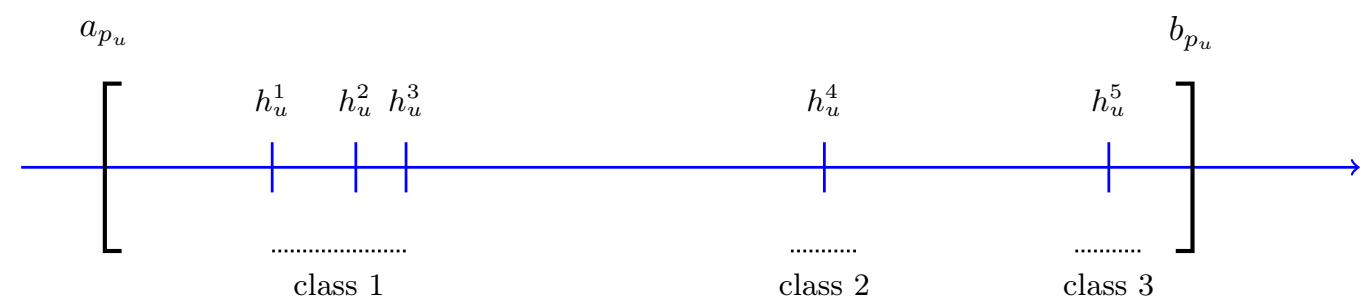

Figure 6: Initial schedule of user $u$

Assume that, for any reason, the value of $h_{u}^{5}$ has to be modified. The value $h_{u}^{5}$ is first removed from the user's schedule. The remaining partial set of service times is $\mathcal{H}_{u} \backslash\left\{h_{u}^{5}\right\}=\left\{h_{u}^{1}, h_{u}^{2}, h_{u}^{3}, h_{u}^{4}\right\}$. It can be observed that the number of time classes of user $u$ is decreased by 1 if the service time $h_{u}^{5}$ is removed, i.e. $\mu\left(\mathcal{H}_{u} \backslash\left\{h_{u}^{5}\right\}\right)=\mu\left(\mathcal{H}_{u}\right)-1$. 
Then, depending on the future value of $h_{u}^{5}$, user $u$ will have two or three time classes. This is illustrated by Figure 7. Any value of $h_{u}^{5}$ in the interval $\left[h_{u}^{3}-\Lambda, h_{u}^{1}+\Lambda\right]$ is compatible with class 1 . Similarly, any value of $h_{u}^{5}$ in the interval $\left[h_{u}^{4}-\Lambda, h_{u}^{4}+\Lambda\right]$ is compatible with class 2. Any value out of these intervals creates a class 3 .

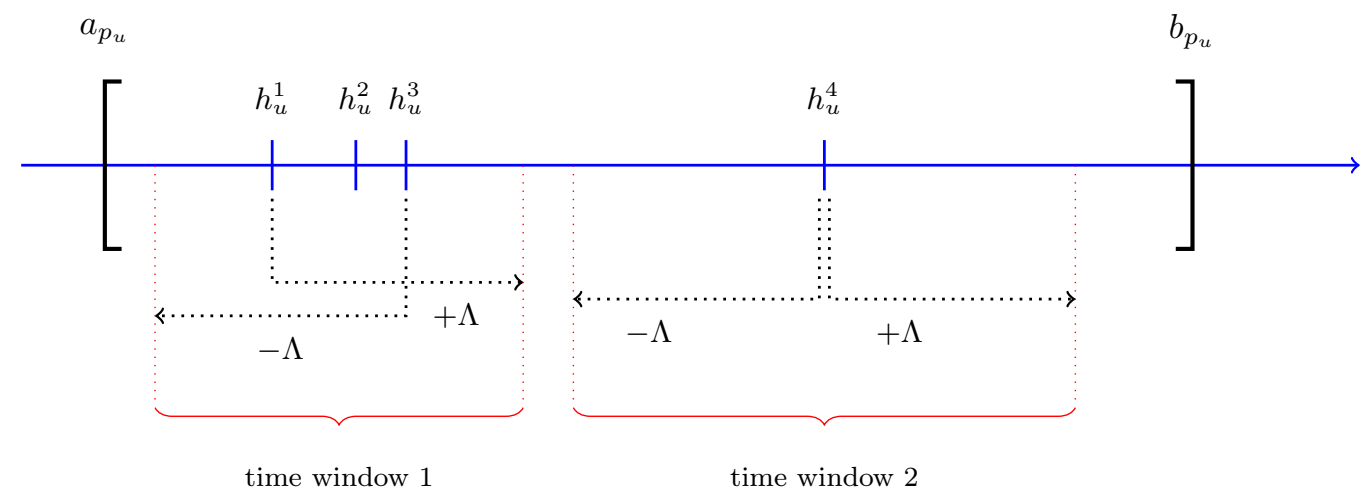

Figure 7: Construction of time windows of user $u$ with $\mathcal{H}=\left\{h_{u}^{1}, h_{u}^{2}, h_{u}^{3}, h_{u}^{4}\right\}$.

As a consequence, if the objective is to have at most two time classes for user $u$, the value of $h_{u}^{5}$ must be defined within times windows $\left[h_{u}^{3}-\Lambda, h_{u}^{1}+\Lambda\right]$ or $\left[h_{u}^{4}-\Lambda, h_{u}^{4}+\Lambda\right]$. If the objective is to have at most three time classes, $h_{u}^{5}$ can take any value in the interval $\left[a_{p_{u}}, b_{p_{u}}\right]$.

Given a user $u$ and a partial set $\mathcal{H}_{u}$ of service times, we call genMTW( $\left.\mathcal{H}_{u}, u\right)$ the function that returns a set of multiple time windows. The algorithm implementing the construction of multiple time windows is detailed in Appendix A.1.

\subsection{Multiple time windows for the DARPmTW}

Section 5.1 presented an example of multiple time windows definition for some user $u$ given a known set ${ }_{460}$ of service times $\mathcal{H}_{u}=\left\{h_{u}^{1}, h_{u}^{2}, h_{u}^{3}, h_{u}^{4}\right\}$. In this section, we define multiple time windows, denoted by $\mathcal{W}_{u}^{t}$, for any user $u \in \mathcal{U}$ and for any set $\mathcal{H}_{u}$. More precisely, we explain how to define the parameters of the function $\operatorname{gen} M T W\left(\mathcal{H}_{u}, u\right)$.

In order to increase consistency and to generate routes that were not in the previous pool $\mathcal{L}$, we propose a very large neighborhood operator that sequentially removes the value of the variables associated with

465 each time period and rebuilds a feasible solution. At each time period, the operator focuses on a particular user, denoted by $\bar{u}$, which is randomly selected among all users with $C_{\max }$ time classes. The idea is to rebuild a solution that decreases the number $\mu\left(\mathcal{H}_{\bar{u}}\right)$ of time classes of user $\bar{u}$ and controls the number $\mu\left(\mathcal{H}_{u}\right)$ of time classes for all other users.

Given one period $t \in \mathcal{T}$, the current value of variables $h_{u}^{t}$ is "forgotten" (removed) and multiple time windows $\mathcal{W}_{u}^{t}$ are set according to the four cases represented in Table 5 . These cases are defined according to the answer to the following questions: i) Is the selected user $\bar{u}$ ? ii) Is the number of time classes of user $u$ decreased by 1 when the service time $h_{u}^{t}$ at period $t$ is removed? This question can be answered by checking if the inequality $\mu\left(\mathcal{H}_{u} \backslash\left\{h_{u}^{t}\right\}\right)<\mu\left(\mathcal{H}_{u}\right)$ holds.

The yes/no answers to these questions yield four ways to define multiple time windows, representing by the four main cells of Table 5 .

Note that using $\mathcal{W}_{u}^{t}=\left\{\left[a_{p_{u}}, b_{p_{u}}\right]\right\}$ in the bottom right-hand case may result in an increase of time classes for user $u$. We observed that this temporary relaxation helps decrease the number of time classes of user $\bar{u}$. The idea of relying on a randomly selected user $\bar{u}$ was originally proposed by [9] to solve a time consistent VRP. The contribution is to extend the approach of [9] from a VRP to a DARP. Finding the best routes that satisfy the multiple time windows amounts to solve a Dial-A-Ride Problem with multiple Time Windows (DARPmTW).

Algorithm 3 describes the process used to generate new routes. It focuses on a randomly selected user $\bar{u}$ with $C_{\max }$ time classes (lines 2). For each time period $t \in \mathcal{T}$, multiple time windows for user $\bar{u}$ are sought such that its number of time classes will be decreased (line 4). For all other users, multiple time windows are sought such that their number of time classes will be controlled (line 6), as detailed in Table 5. These time windows are used to define a new instance of the DARPmTW for each time period. Solving these instances yields a set of routes denoted by $\mathcal{L}_{\text {new }}^{t}$ for each time period $t$ (line 8). The sets 


\begin{tabular}{|c|c|c|}
\hline \multicolumn{3}{|c|}{$\begin{array}{c}\text { Is the number of time classes of user } u \text { decreased by } 1 \text { when the } \\
\text { service time } h_{u}^{t} \text { at period } t \text { is removed? }\end{array}$} \\
\hline & Yes & No \\
\hline 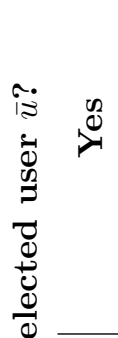 & $\begin{array}{l}\text { Changing the service time } h_{\bar{u}}^{t} \text { to any } \\
\text { service time in the time windows } \mathcal{W}_{\bar{u}}^{t} \\
\text { has to decrease the number of time } \\
\text { classes for this user: } \\
\qquad \mathcal{W}_{\bar{u}}^{t}=\operatorname{genMTW}\left(\mathcal{H}_{\bar{u}} \backslash\left\{h_{\bar{u}}^{t}\right\}, \bar{u}\right) .\end{array}$ & $\begin{array}{l}\text { Let }\left[e_{\bar{u}}^{t}, l_{\bar{u}}^{t}\right] \in \mathcal{W}\left(\mathcal{H}_{\bar{u}}, \bar{u}\right) \text { be the time } \\
\text { window satisfied by service time } h_{\bar{u}}^{t} \\
\left(e_{\bar{u}}^{t} \leq h_{\bar{u}}^{t} \leq l_{\bar{u}}^{t}\right) \text {. Defining } \\
\qquad \mathcal{W}_{\bar{u}}^{t}=\operatorname{genMTW}\left(\mathcal{H}_{\bar{u}}, \bar{u}\right) \backslash\left\{\left[e_{\bar{u}}^{t}, l_{\bar{u}}^{t}\right]\right\} \\
\text { will enforce a decrease in the number of } \\
\text { time classes for this user. }\end{array}$ \\
\hline 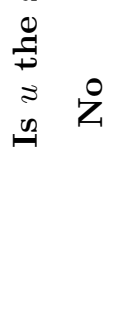 & $\begin{array}{l}\text { The service time } h_{u}^{t} \text { defines a time class } \\
\text { on its own and any feasible service time } \\
\text { at period } t \text { can be accepted: } \\
\qquad \mathcal{W}_{u}^{t}=\left\{\left[a_{p_{u}}, b_{p_{u}}\right]\right\}\end{array}$ & $\begin{array}{l}\mathcal{W}_{\bar{u}}^{t}=\operatorname{genMTW}\left(\mathcal{H}_{\bar{u}}, \bar{u}\right) \backslash\left\{\left[e_{\bar{u}}^{t}, l_{\bar{u}}^{t}\right]\right\}, \\
\text { with a probability } \nu=\theta \times\left(C_{\max }-\right. \\
\left.\mu\left(\mathcal{H}_{u}\right)\right), \text { where } \theta \text { is a fixed parameter. } \\
\text { Otherwise, } \mathcal{W}_{u}^{t}=\left\{\left[a_{p_{u}}, b_{p_{u}}\right]\right\} .\end{array}$ \\
\hline
\end{tabular}

Table 5: Overview of cases for the definition of multiple time windows $\mathcal{W}_{u}^{t}$.

$\mathcal{L}_{\text {new }}^{t}$ are finally merged (line 10 and returned. In section 5.3 we detail the solution method used to solve the DARPmTW instances (line 8).

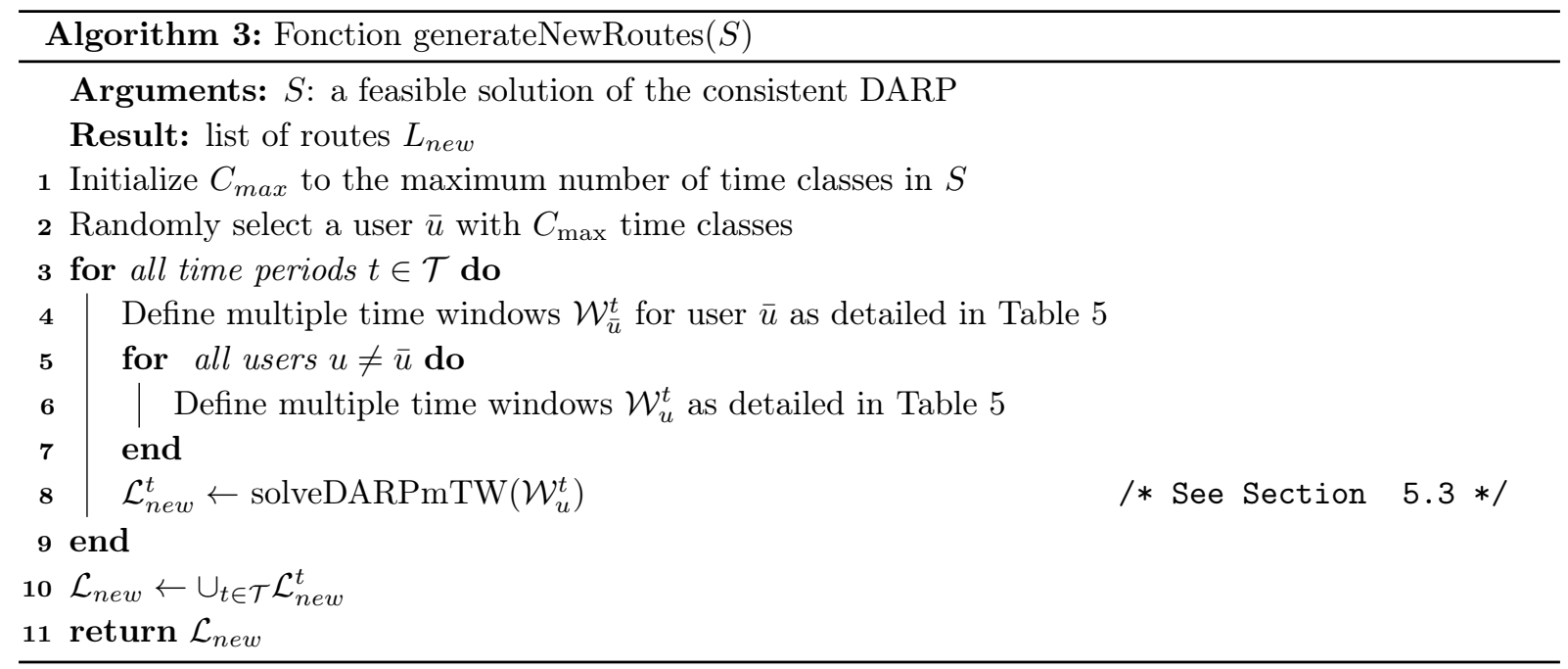

Line 8 of Algorithm 3 consists of solving a DARP with multiple Time Windows (DARPmTW). Additionally, the duration of routes should be minimized: waiting times caused by time windows are authorized but waiting times aimed at artificially improving time consistency are forbidden.

To solve this optimization problem, we reuse the LNS-SCP used in Section 4.3 to solve single period DARPs with one time window at each pickup or delivery point. However, this LNS-SCP requires two adaptations. First, since the SCP component is somewhat redundant with the set partitioning problems solved in $\mathrm{SP} \varepsilon \mathrm{C}$, we do not activate it here. The algorithm is therefore called LNS in the following. Second, a key point of the LNS is to handle the route duration minimization when repairing destroyed solutions. This amounts to check a set of temporal constraints through a route scheduling algorithm, called the DARPmTW scheduling algorithm.

More specifically, let us consider the feasibility check of an insertion in a route $\omega \in \Omega$. For each user 
$u$ visited by the route $\omega$, the DARPmTW scheduling algorithm checks temporal constraints: a maximum ride time $\bar{T}_{u}$, a pickup time window $\left[a_{p_{u}}, b_{p_{u}}\right]$ and a delivery time window $\left[a_{d_{u}}, b_{d_{u}}\right]$. If these temporal constraints are satisfied, a service time $h_{i}$ is computed for each node such that: (i) the route duration is minimized and (ii) service times are scheduled as early as possible at each pickup node, delivery node or depot. The DARPmTW scheduling algorithm computes a maximum route time shift $\Delta_{\omega}^{+}$. This value indicates how much the route schedule can be shifted forward, while preserving both its feasibility and the route duration. Hence, shifting the starting time of a route by a value $0 \leq \delta \leq \Delta_{\omega}^{+}$shifts the service times of all vertices on the route by the same value without impacting the route duration nor waiting times. The detailed procedure, proposed in Tellez et al. [37], can be found in Appendix A.2.

Once the pickup time window $\left[a_{p_{u}}, b_{p_{u}}\right]$, the delivery time window $\left[a_{d_{u}}, b_{d_{u}}\right]$ and ride time constraints $\bar{T}_{u}$ are satisfied, we check the satisfaction of the multiple time windows $\mathcal{W}_{u}^{t}$ with a new procedure. Specifically, this procedure checks if there is a service time for each user $u$ compatible with a time window in $\mathcal{W}_{u}^{t}$. Given a route $\omega \in \Omega$ with a minimal duration and service times scheduled as early as possible, Algorithm 4 checks if each user $u$ on this route can be picked up within a set of multiple time windows $\mathcal{W}_{u}^{t}$ without increasing the duration of the route. Accordingly, the only variable in this algorithm is the departure time of the route, which can be postponed by a value $0 \leq \delta \leq \Delta_{\omega}^{+}$called route time shift. Note that, for morning routes, the service in multiple time windows is only verified at pickups because time consistency is measured at these nodes. For afternoon routes, time consistency must be checked at delivery points only.

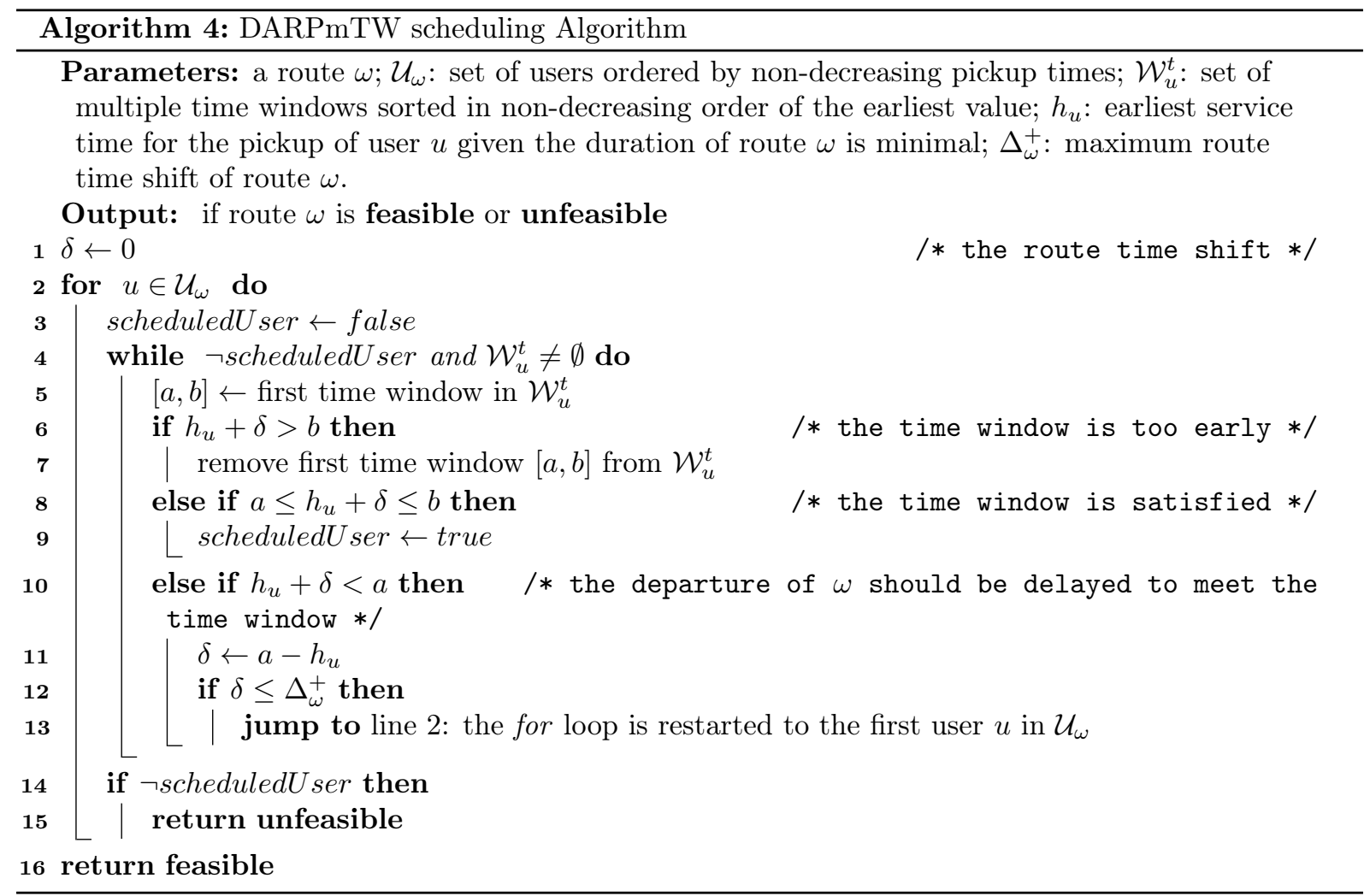

Let $\mathcal{U}_{\omega}$ be the set of users ranked in non-decreasing order of their pickup service time. Algorithm 4 looks for a route time shift $\delta \in\left[0, \Delta_{\omega}^{+}\right]$such that for all users $u \in \mathcal{U}_{\omega}$ there exists a time window $[a, b] \in \mathcal{W}_{u}^{t}$ in which the shifted service time $h_{u}+\delta$ can be scheduled. Note that all nodes in route are shifted forward by the same quantity of time. Thus, it is not possible to increase the duration of the route in order to ensure feasibility.

In line 1, the route time shift $\delta$ is initialized to 0 . Users are considered sequentially (line 2). For each user $u \in \mathcal{U}$, its first time window $[a, b]$ is evaluated (lines 4,5 ). Three cases are considered:

(i) If the shifted service time takes place after the end of the time window $\left(h_{u}+\delta>b\right)$, the time window $[a, b]$ can never be satisfied: it is removed from set $\mathcal{W}_{u}^{t}$ (lines 6 7). The next iteration of the while loop will directly check the next time window for user $u$.

(ii) If the shifted service time takes place in time window $[a, b]$, the shifted service time is feasible for 
the pickup of user $u$ (lines 8,9). The algorithm continues with the next user.

(iii) If the shifted service time takes place before the opening of the time window $\left(h_{u}+\delta<a\right.$, line 10 , the route shift $\delta$ has to be increased to $a-h_{u}$, so that the new shifted service time is exactly $a$ (line 11). At this point, two cases are possible a) the new value of route time shift $\delta$ is feasible (i.e. $\delta \leq \Delta_{\omega}^{+}$), and the main loop is restarted from the first user with the new value of $\delta$ (jump from line 13 to line 22; b) $\delta$ is larger than the maximum allowed shift $\Delta_{\omega}^{+}$and the route is infeasible (line 15).

Finally, if each user has a feasible time window given the same time shift $\delta$, then the route is declared 540 feasible (line 16 ).

In the worst case, all time windows are removed $\left(\sum_{u \in \mathcal{U}_{\omega}}\left|\mathcal{W}_{u}^{t}\right|\right.$ operations). For each removal, the route time shift is increased and then the procedure is restarted. One iteration of the main loop (2, 13) cannot take more than $\left|\mathcal{U}_{\omega}\right|$ operations. So the worse-case time complexity of Algorithm 4 is $\mathcal{O}\left(\left|\overrightarrow{\mathcal{U}}_{\omega}\right| \times\right.$ $\left.\sum_{u \in \mathcal{U}_{\omega}}\left|\mathcal{W}_{u}^{t}\right|\right)$

\section{sas 6 Computational experiments}

The matheuristic described in Section 4.1 was coded in $\mathrm{C}++$ and the mathematical models were solved with CPLEX Concert Technology 12.6 running on a single thread on an Intel Xeon E5-1620 v3 @3.5Ghz processor.

This section details computational experiments in two families of instances. It is structured as follows: Section 6.1 presents the value of parameters used by our algorithms. Section 6.2 introduces the instances used to evaluate our approach. They are built from real data provided by the Synergihp Rhône-Alpes Company. In Section 6.3. SPEC is assessed on benchmark instances of [1] and $[9]$ for the time consistent VRP. Finally, Section 6.4 presents managerial insights regarding cost performance and time consistency.

\subsection{Parameter settings}

After preliminary tests on a representative subset of instances, parameters shown in Table 6 were found to provide the best average performance.

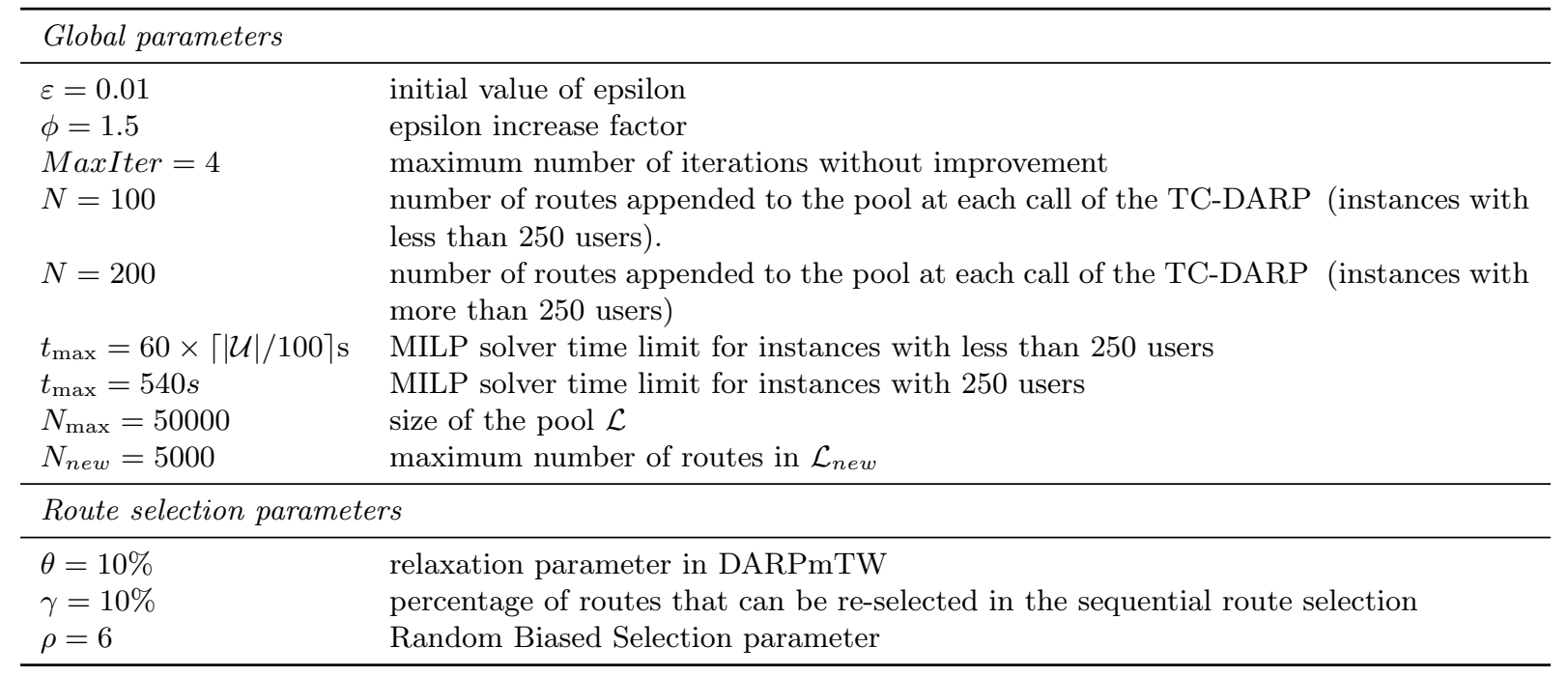

Table 6: Summary of parameters of the SPEC algorithm

The value of parameter $\varepsilon$ has a strong impact on the computing time. Higher values of $\varepsilon$ help to reduce the computation time. However, the quality of the Pareto front approximation is considerably deteriorated. Thus, $\varepsilon=0.01$ was taken as a good trade-off between computing time and quality of the solution. $\mathrm{SP} \varepsilon \mathrm{C}$ is less sensitive to parameter $\phi$ but its value needs to be greater than 1.5 to have significant impact on the value of $\varepsilon$.

We found that a value of MaxIter greater than 4 does not improve the quality of each point of the Pareto front approximation. Parameters $N$ and $t_{\max }$ were determined in order to maximize the number of times when the MILP solver is able to solve the TC-DARPs to proven optimality. 
The TC-DARP considers two pools of routes: $\mathcal{L}$ and $\mathcal{L}_{\text {new }}$. In order to keep the number of routes in memory under control, limits in the maximum size of the pool $\mathcal{L}$ and of the $\mathcal{L}_{\text {new }}$ were set to $N_{\text {max }}=50000$ and $N_{\text {new }}=5000$, respectively.

We compared several settings of the algorithm in order to assess its key components. In particular, we run a variant of the $\mathrm{SP} \varepsilon \mathrm{C}$ algorithm without the procedure presented in Section 4.4 to generate new routes. This resulted in a slight cost increase on solutions with 2 time classes and more, and a $10 \%$ cost increase on solutions with 1 time class. Moreover, the hypervolume indicator increases by $17 \%$ if the generation of new routes is disabled.

We also implemented a randomized route selection based on the roulette wheel mechanism inspired by 26]. This randomization did not bring significant improvement. A detailed evaluation of the algorithm's components can be found in 36 .

\subsection{Description of instances}

The time consistent DARP studied in this paper arises in the context of transportation of people with disabilities. We collected real data from the Synergihp Rhône-Alpes Company based in Lyon, France. This data concerns the transportation of hundreds of users to MSIs.

From Monday to Friday, the users are carried in the morning from their home to a MSI. In the afternoon, they are driven back home. Without loss of generality, this paper presents the results of our research for morning trips.

The data collected from Synergihp includes 575 users from a large geographical area around the city of Lyon. We partitioned this data in two independent geographical areas (distinct users, distinct MSIs), yielding two large instances with with 280 to 295 users, respectively. These two large instances were in turn partitioned into 4 medium-size instances with 120 to 160 users. Finally, the four mediumsize instances were partitioned into 8 small-size instances with 60 to 80 users. We assume an infinite homogeneous fleet of vehicles with a capacity of 4 seats and 3 wheelchair spaces. Vehicle costs provided by the company include an hourly cost $\alpha=23.8 €$ and a cost per kilometer $\tau=0.17 €$. No fixed vehicle cost $\lambda$ was given by the company. Moreover, with a homogeneous fleet of vehicles, this cost has no major impact on the solution nor on solutions consistency. Thus, we simply assume an arbitrarily small value $\lambda=1$ in order to favor solutions with similar route cost but fewer vehicles.

Travel times and distances are obtained from the Open Source Routing Machin ${ }^{2}$ (OSRM) proposed by Luxen and Vetter [20]. For each user $u \in \mathcal{U}$, we define maximum ride times according to direct travel time $t_{p_{u}, d_{u}}$ between the pickup location $p_{u}$ and the delivery location $d_{u}$. The following formula generates maximum ride times $(R T)$ that are between 15 and 30 minutes longer than direct travel times: $R T=15 \times\left\lceil\left(t_{p_{u}, d_{u}}+15\right) / 15\right\rceil$.

Time windows at MSIs are 15 minutes wide. The size of time classes is 10 minutes wide. Finally, time windows at pickup locations and service times strongly influence the actual design of routes, but they have no impact on the efficiency of our solution method. We therefore ignored them for the sake of simplicity.

\subsection{Performance evaluation on benchmarks from the literature}

As the TC-DARP is a new problem, there is no benchmark in the literature. However, to evaluate the performance of $\mathrm{SP} \varepsilon \mathrm{C}$, we solve reference instances for two other time consistent routing problems. The first benchmark is an adaptation of the conVRP instances of Groër et al. 11. This adaptation, proposed by Feillet et al. [9], transforms the small conVRP instances of Groër et al. 11] into TC-VRP instances. These instances are denoted RconVRP. They include up to 12 users over 3 days and have been solved to optimality by a MILP solver. The second benchmark set is the TC-VRP from Feillet et al. [9. It contains instances for up to 65 users over 5 time periods.

Although the TC-VRP is the closest problem to the TC-DARP, there are some differences between both problems. The TC-VRP has the following hypothesis: (i) it has a single depot and no time windows; (ii) it assumes a limited fleet of vehicles; (iii) the consistency objective function is the maximal number of time classes over all users (i.e. $C_{\max }$ ); and (iv) routes must start at time 0 , with no waiting time allowed. To be solved by SP $\varepsilon$ C, TC-VRP instances have been converted to TC-DARP instances by defining one

615 copy of the depot for each request. Ride times and time windows have been relaxed by setting arbitrary large values. Finally, since the VRP routes are not subject to time windows nor ride times, routes can

2 http://project-osrm.org/ 
be traveled in either direction. Thus, each time a route is appended to the pool, the reverse route is also appended.

\subsubsection{RconVRP instances}

This benchmark proposes 10 small instances of the TC-VRP: the first 5 instances with 10 users and the next 5 with 12 users. Instances have been solved to optimality with CPLEX. Table 7 presents the comparison with $\mathrm{SP} \varepsilon \mathrm{C}$ over 10 runs.

\begin{tabular}{r|rrr|rrr}
\hline Instance & \multicolumn{3}{c}{ Opt. Cost } \\
\cline { 2 - 7 }$C_{\max }$ & \multicolumn{1}{c}{$\leq 3$} & \multicolumn{1}{c}{$\leq 2$} & \multicolumn{1}{c}{1} & \multicolumn{1}{c}{$\leq 3$} & \multicolumn{1}{c}{$\leq 2$} & \multicolumn{1}{c}{1} \\
\hline RconVRP10-1 & 92.91 & 92.91 & 92.91 & $\mathbf{9 2 . 9 1}$ & $\mathbf{9 2 . 9 1}$ & $\mathbf{9 2 . 9 1}$ \\
RconVRP10-2 & 80.42 & 80.42 & 80.96 & $\mathbf{8 0 . 4 2}$ & $\mathbf{8 0 . 4 2}$ & 82.83 \\
RconVRP10-3 & 94.12 & 94.12 & 94.37 & $\mathbf{9 4 . 1 2}$ & $\mathbf{9 4 . 1 2}$ & $\mathbf{9 4 . 3 7}$ \\
RconVRP10-4 & 93.71 & 93.71 & 94.09 & $\mathbf{9 3 . 7 1}$ & $\mathbf{9 3 . 7 1}$ & $\mathbf{9 4 . 0 9}$ \\
RconVRP10-5 & 83.84 & 83.84 & 96.01 & $\mathbf{8 3 . 8 4}$ & 84.50 & 96.70 \\
RconVRP12-1 & 103.65 & 103.65 & 104.40 & $\mathbf{1 0 3 . 6 5}$ & $\mathbf{1 0 3 . 6 5}$ & 109.19 \\
RconVRP12-2 & 73.89 & 73.89 & 81.25 & $\mathbf{7 3 . 8 9}$ & $\mathbf{7 3 . 8 9}$ & 83.40 \\
RconVRP12-3 & 82.77 & 82.77 & 83.12 & $\mathbf{8 2 . 7 7}$ & $\mathbf{8 2 . 7 7}$ & $\mathbf{8 3 . 1 2}$ \\
RconVRP12-4 & 97.57 & 97.57 & 101.91 & $\mathbf{9 7 . 5 7}$ & 98.55 & 104.31 \\
RconVRP12-5 & 83.63 & 83.63 & 89.25 & $\mathbf{8 3 . 6 3}$ & $\mathbf{8 3 . 6 3}$ & 91.38 \\
\hline
\end{tabular}

Table 7: Benchmark on RconVRP instances reported in 9

Columns 2-4 (Opt Cost), present the cost of optimal solutions for each time class. In the next 3 columns, we report the average performance of SPEC for each time class. Note that SPEC could not find most of the solutions with 3 time classes and one with 2 time classes. For each instance, the Gap is computed as (Avg Cost - Best) / Best $\times 100$. The last row is the average gap (Avg Gap) overall instances which is the average value of the corresponding column.

For single time class solutions, SPEC founds optimal solutions in the 10 runs for 4 instances of 10. The average gap is $1.5 \%$. The performance is better with 2 time classes for which 7 of 10 solutions are optimal and with an average gap of $0.2 \%$.

\subsubsection{Results on time consistent VRP instances}

This benchmark was built from real data collected in 14 distinct MSIs, with a number of users ranging from 15 to 65, and a number of time periods equal to 5 (Monday to Friday). For each MSI, 5 profiles of transportation demands where randomly generated where each profile corresponds to the percentage of

${ }_{635}$ people present during the complete week. This percentage varies between $50 \%$ and $90 \%$. This yields a total of 70 benchmark instances.

Transportation cost of solutions with 1 to 5 time classes are provided for most instances. Feillet et al. 9] solved the TC-VRP with an LNS-based matheuristic with a time limit of 1 hour. SPEC stops when all users reach a single time class. For each value of $C_{\max }$, our lexicographic optimization explores all

640 non-dominated solutions. This approach is more time consuming but returns a more complete Pareto front approximation that can help decision makers to select intermediate trade-off solutions for each time class.

Tables 8 and 9 show the average gap of $\mathrm{SP} \varepsilon \mathrm{C}$ with respect to the LNS method of Feillet et al. [9], aggregated in two different ways. For each instance, we compute the gap as (Cost SP $\varepsilon$ C - Cost LNS) $/$ Cost LNS $\times$

645 100. Thus, any negative gap represents an improvement.

Table 8 shows the numerical results aggregated by percentage of presence during the week. For example, data-5-Y aggregates instances where, on average, $50 \%$ of users are transported everyday, while in the group data-9-Y the average percentage of users transported rises to $90 \%$.

The average relative gap (Avg Gap) overall instances between the results obtained with SPEC and the LNS was improved for all time classes. However, slightly better results are reported for solutions with 3,4 and 5 time classes. This result is confirmed with the number of new best-known solutions (Nb new BKS) which is more than 50 for solutions above 3 time classes, and 35 for solutions with 1 and 2 time 


\begin{tabular}{lrrrrr}
\hline Instance & \multicolumn{5}{c}{ Avg Gap Transportation cost } \\
\cline { 2 - 6 } \multicolumn{1}{c}{$C_{\max }$} & \multicolumn{1}{c}{$\leq 5$} & \multicolumn{1}{c}{$\leq 4$} & \multicolumn{1}{c}{$\leq 3$} & \multicolumn{1}{c}{$\leq 2$} & \multicolumn{1}{c}{1} \\
\hline data5-Y & $\mathbf{- 1 . 1 0 \%}$ & $\mathbf{- 1 . 0 0 \%}$ & $\mathbf{- 1 . 0 2 \%}$ & $\mathbf{- 0 . 2 1 \%}$ & $\mathbf{- 0 . 3 7 \%}$ \\
data6-Y & $\mathbf{- 1 . 0 3 \%}$ & $\mathbf{- 1 . 0 3 \%}$ & $\mathbf{- 0 . 8 1 \%}$ & $\mathbf{- 0 . 2 6 \%}$ & $0.83 \%$ \\
data7-Y & $\mathbf{- 1 . 0 9 \%}$ & $\mathbf{- 0 . 9 6 \%}$ & $\mathbf{- 0 . 8 6 \%}$ & $0.13 \%$ & $\mathbf{- 0 . 1 3 \%}$ \\
data8-Y & $\mathbf{- 1 . 0 6 \%}$ & $\mathbf{- 1 . 0 0 \%}$ & $\mathbf{- 0 . 7 2 \%}$ & $0.00 \%$ & $\mathbf{- 0 . 2 5 \%}$ \\
data9-Y & $\mathbf{- 0 . 6 1 \%}$ & $\mathbf{- 0 . 6 1 \%}$ & $\mathbf{- 0 . 4 9 \%}$ & $\mathbf{- 0 . 2 7 \%}$ & $\mathbf{- 1 . 7 6 \%}$ \\
\hline Avg Gap (\%) & $\mathbf{- 0 . 9 8 \%}$ & $\mathbf{- 0 . 9 2 \%}$ & $\mathbf{- 0 . 7 8 \%}$ & $\mathbf{- 0 . 1 2 \%}$ & $\mathbf{- 0 . 3 3 \%}$ \\
Nb Sols & 70 & 70 & 70 & 70 & 70 \\
Nb new BKS & 63 & 59 & 53 & 35 & 35 \\
\hline
\end{tabular}

Table 8: Results aggregated by percentage of user requests on the benchmark of Feillet et al. 9]

classes. A total number of 245 strictly new best solutions were found, as shown on the last row of the table. Detailed results can be found in Appendix B

Table 9 shows the numerical results aggregated by MSI. The last two digits of the instance name represent the number of users. This table shows that $\mathrm{SP} \varepsilon \mathrm{C}$ has better performance with larger instances. However, dataX-59 instances are in particular the most difficult to solve for $\mathrm{SP} \varepsilon \mathrm{C}$, with an extra cost of $3.41 \%$ for $C_{\max }=2$ solutions and $2.39 \%$ for $C_{\max }=1$ solutions.

\begin{tabular}{l|rrrrr}
\hline Instance & \multicolumn{5}{c}{ Avg Gap Transportation cost } \\
\cline { 2 - 6 } \multicolumn{1}{c}{$C_{\max }$} & \multicolumn{1}{c}{$\leq 5$} & \multicolumn{1}{c}{$\leq 4$} & \multicolumn{1}{c}{$\leq 3$} & \multicolumn{1}{c}{$\leq 2$} & \multicolumn{1}{c}{1} \\
\hline dataX-15 & $0.03 \%$ & $0.03 \%$ & $\mathbf{0 . 0 0 \%}$ & $0.47 \%$ & $3.04 \%$ \\
dataX-21 & $\mathbf{- 0 . 1 5 \%}$ & $0.07 \%$ & $0.18 \%$ & $0.24 \%$ & $1.53 \%$ \\
dataX-25 & $\mathbf{- 0 . 2 1 \%}$ & $\mathbf{- 0 . 0 6 \%}$ & $0.01 \%$ & $0.19 \%$ & $0.53 \%$ \\
dataX-26 & $\mathbf{- 0 . 2 1 \%}$ & $\mathbf{- 0 . 2 1 \%}$ & $\mathbf{- 0 . 0 3 \%}$ & $0.04 \%$ & $0.08 \%$ \\
dataX-27 & $\mathbf{- 0 . 2 7 \%}$ & $\mathbf{- 0 . 1 2 \%}$ & $\mathbf{- 0 . 1 6 \%}$ & $0.03 \%$ & $\mathbf{- 0 . 9 3 \%}$ \\
dataX-32 & $\mathbf{- 0 . 5 4 \%}$ & $\mathbf{- 0 . 5 4 \%}$ & $\mathbf{- 0 . 1 9 \%}$ & $0.10 \%$ & $0.49 \%$ \\
dataX-41 & $\mathbf{- 1 . 1 5 \%}$ & $\mathbf{- 1 . 1 5 \%}$ & $\mathbf{- 1 . 6 5 \%}$ & $\mathbf{- 1 . 1 9 \%}$ & $\mathbf{- 2 . 4 6 \%}$ \\
dataX-44 & $\mathbf{- 0 . 7 5 \%}$ & $\mathbf{- 0 . 7 5 \%}$ & $\mathbf{- 0 . 6 1 \%}$ & $\mathbf{- 0 . 7 2 \%}$ & $\mathbf{- 1 . 1 9 \%}$ \\
dataX-46 & $\mathbf{- 1 . 0 5 \%}$ & $\mathbf{- 1 . 0 5 \%}$ & $\mathbf{- 0 . 6 2 \%}$ & $\mathbf{- 0 . 1 8 \%}$ & $\mathbf{- 0 . 3 4 \%}$ \\
dataX-48 & $\mathbf{- 1 . 2 4 \%}$ & $\mathbf{- 0 . 9 8 \%}$ & $\mathbf{- 0 . 7 7 \%}$ & $\mathbf{- 0 . 2 0 \%}$ & $\mathbf{- 1 . 3 8 \%}$ \\
dataX-55 & $\mathbf{- 1 . 9 1 \%}$ & $\mathbf{- 1 . 9 1 \%}$ & $\mathbf{- 1 . 6 3 \%}$ & $\mathbf{- 0 . 9 2 \%}$ & $\mathbf{- 4 . 8 4 \%}$ \\
dataX-59 & $\mathbf{- 2 . 1 7 \%}$ & $\mathbf{- 2 . 1 2 \%}$ & $\mathbf{- 1 . 6 2 \%}$ & $3.41 \%$ & $2.39 \%$ \\
dataX-64 & $\mathbf{- 2 . 3 0 \%}$ & $\mathbf{- 2 . 3 0 \%}$ & $\mathbf{- 2 . 1 4 \%}$ & $\mathbf{- 1 . 6 6 \%}$ & $\mathbf{- 0 . 1 8 \%}$ \\
dataX-65 & $\mathbf{- 1 . 7 7 \%}$ & $\mathbf{- 1 . 7 9 \%}$ & $\mathbf{- 1 . 7 1 \%}$ & $\mathbf{- 1 . 3 3 \%}$ & $\mathbf{- 1 . 4 2 \%}$ \\
\hline Avg Gap $\%)$ & $\mathbf{- 0 . 9 8 \%}$ & $\mathbf{- 0 . 9 2 \%}$ & $\mathbf{- 0 . 7 8 \%}$ & $\mathbf{- 0 . 1 2 \%}$ & $\mathbf{- 0 . 3 3 \%}$ \\
\hline
\end{tabular}

Table 9: Results aggregated by instance size on 9 benchmark

\subsection{Managerial insights on time consistency and transportation costs}

This section reports managerial insights regarding the relationship between time consistency and transportation costs. Figures $8[10$ show the Pareto front approximation obtained on Synergihp Rhône-Alpes instances with 60, 160 and 280 users, respectively. The transportation cost is presented as the percentage of cost increase with respect to the cheapest solution found in that instance (x-axis). The time consistency of non-dominated solutions is shown in a vector form on the vertical axis. Each element of the vector represents the number of users with 3,2 and 1 time classes on that solution, respectively. Note that solutions with 4 or 5 time classes are not represented because, in our tests, they have always been dominated by a solution with 3 time classes.

These Pareto front approximations provide decision makers with a fine intuition of the cost of time consistency associated with each user. The first finding is that all Pareto front approximations start with the majority of users having a single time class and very few users having 3 time classes. With a minor increase of cost, all users have at most 2 time classes (until the dotted line). This means that an useful 


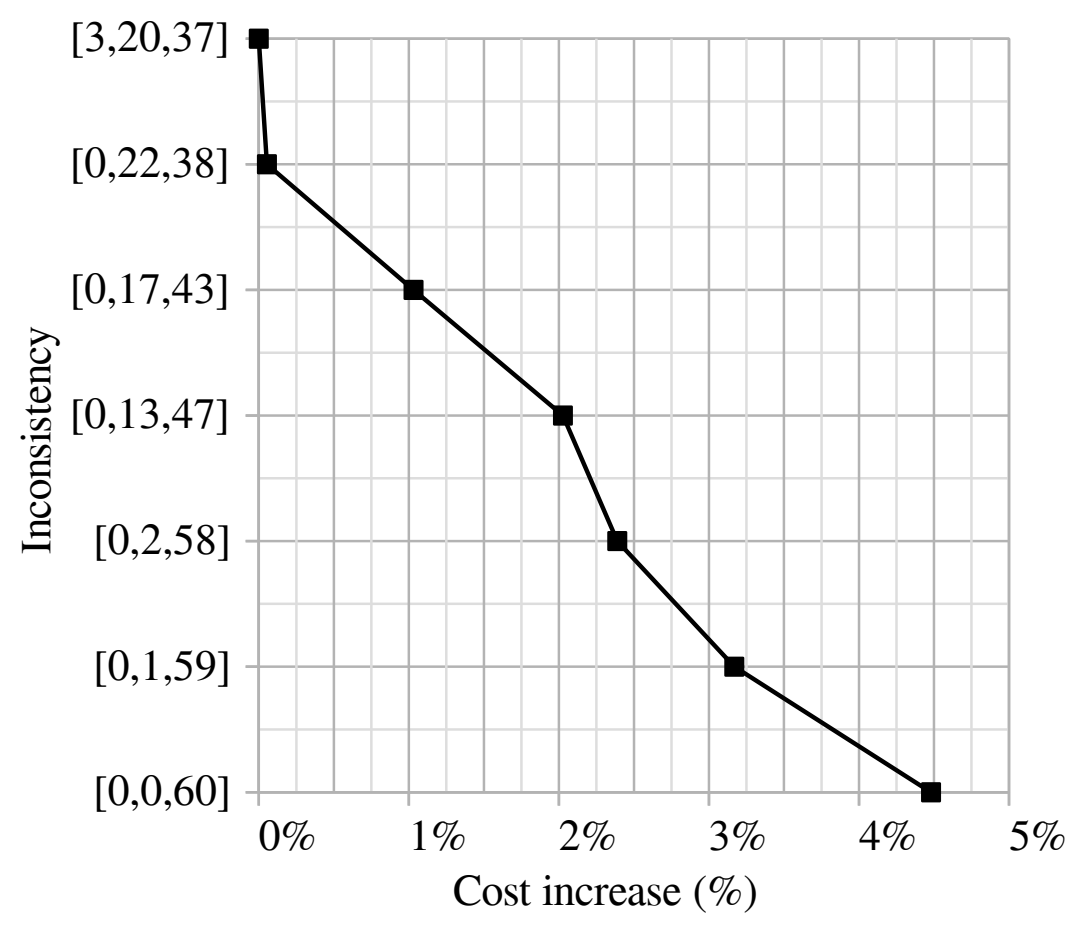

Figure 8: Pareto front approximation for instance TCDARP $\_01 \_60$

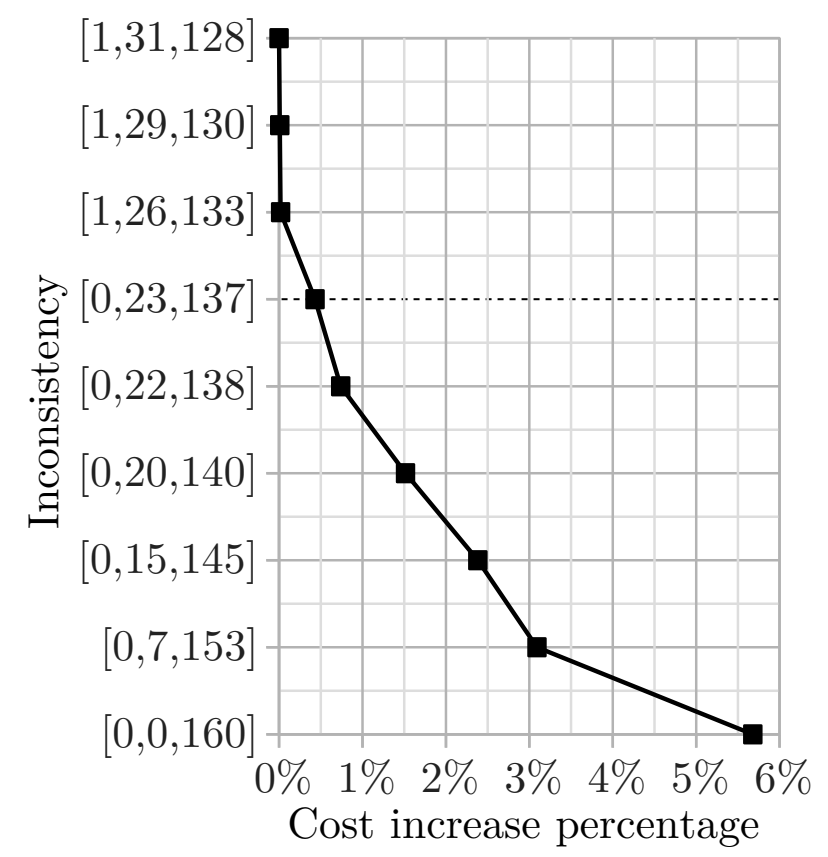

Figure 9: Pareto front approximation for instance TCDARP_10_160 


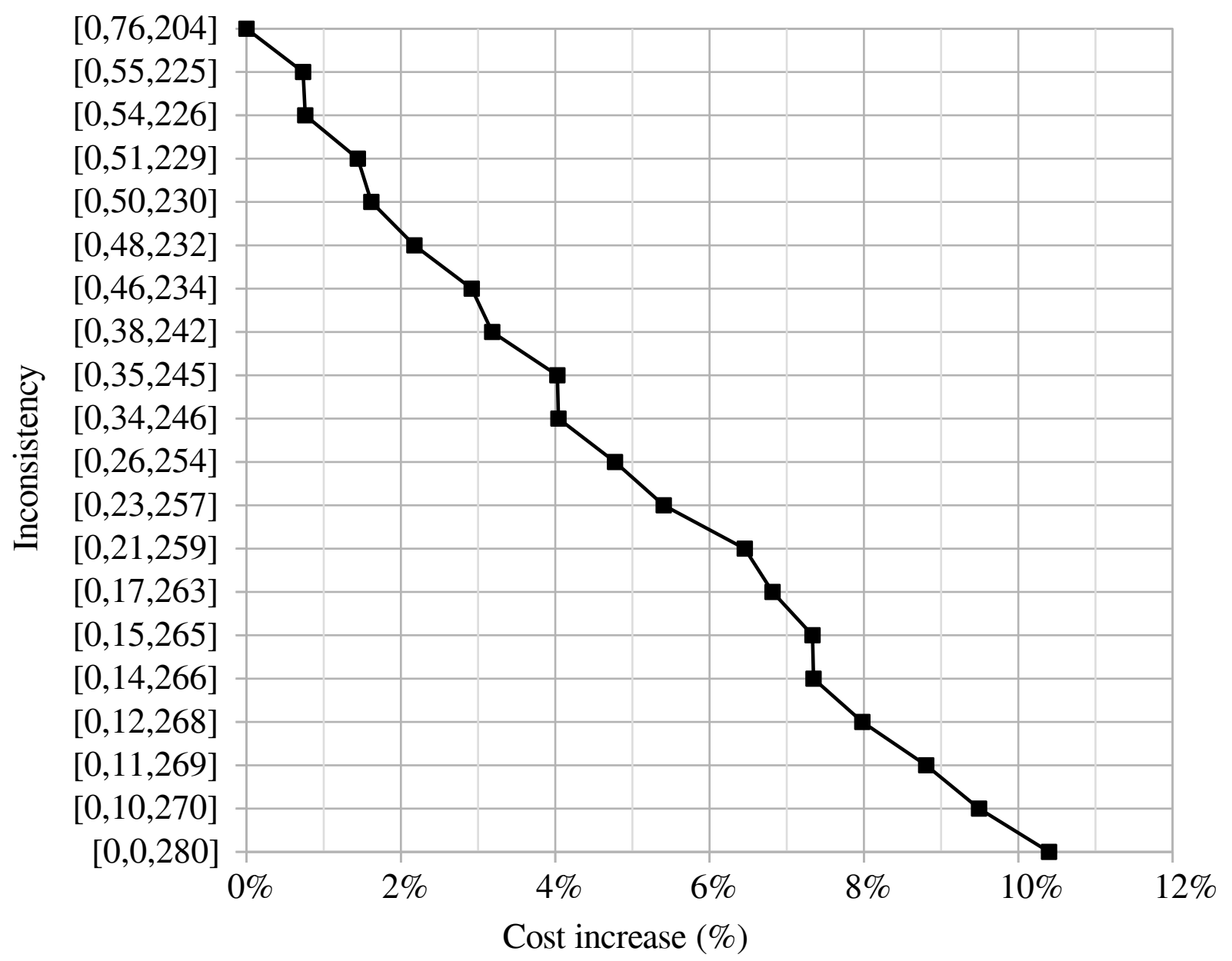

Figure 10: Pareto front approximation for instance TCDARP_12_280 
consistent solution can be found with respect to the cost of the cheapest solution, and a small increase of cost can significantly improve the solution for users with many time classes.

Depending on the instance, the increase of cost for reaching single time class solutions can vary from $1 \%$ to $10 \%$. Note that values on the y-axis are ordered but non-scaled as the distance between points is always constant. Figure 11 shows the same instances on a common scale for solutions with a maximum of 2 time classes per user. The y-axis presents the percentage of users with 2 time classes. It shows that each instance has very different trade-offs depending on the size and the consistency level of the solution.

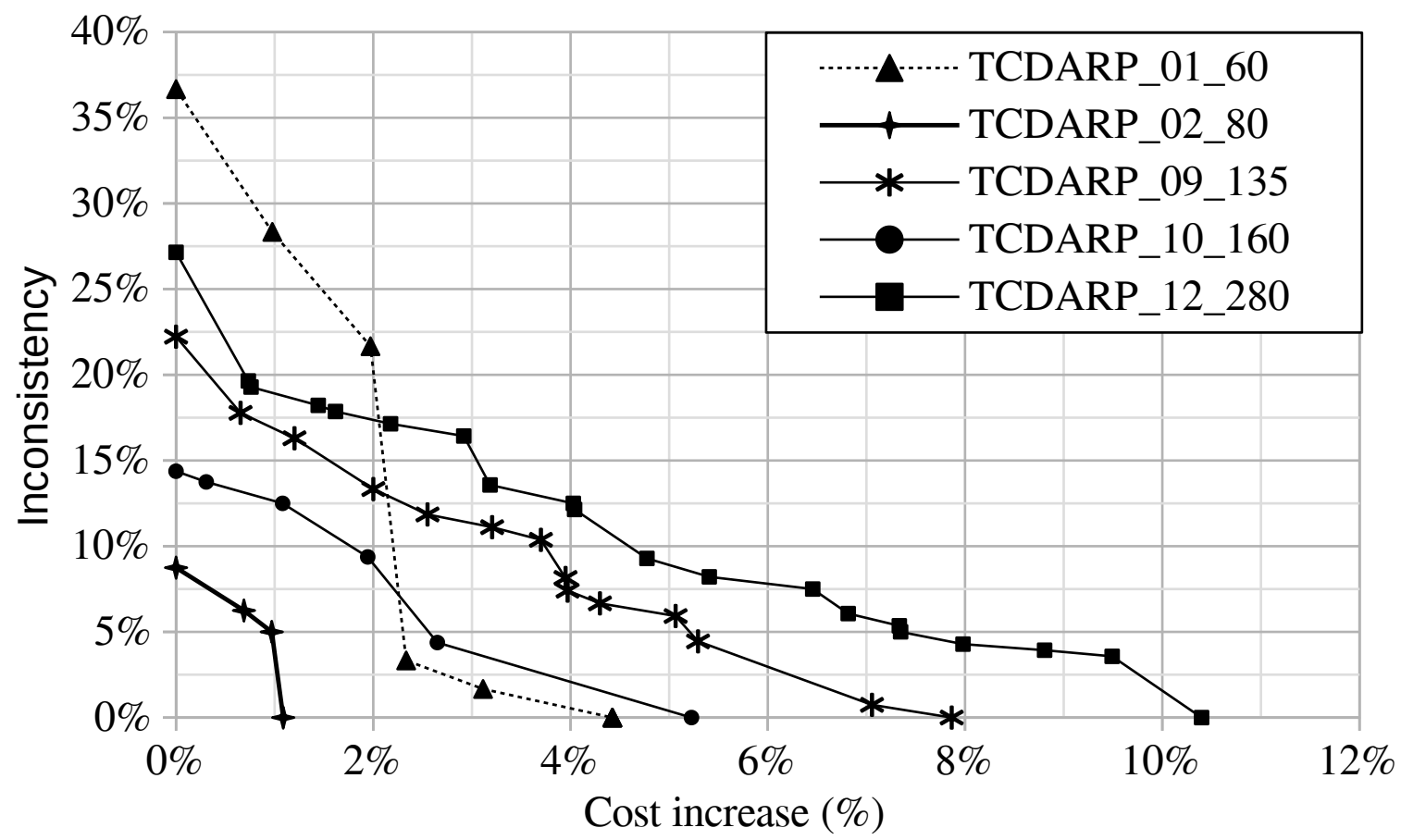

Figure 11: Scaled representation of the Pareto front approximations on five representative instances

Table 10 gives some additional details on the non-dominated solutions shown in Figure 8 (instance TCDARP_01_60). The first column reports the inconsistency vector of each non-dominated solution. Columns $\overline{2}$ to $\overline{4}$ show the cost, the average route duration and the average ride time per user in the corresponding solutions, respectively. The vectors in column 5 represent the number of routes for each time period (from Monday to Friday). The last column reports the total number of routes. This example shows that improving consistency requires increasing the number of routes, which results in a decrease of the average route duration. It is noticeable that the number of routes for a given day does not increase monotonously. For example, the number of routes on Wednesdays is 3 in the first two solutions, then 5 and finally 4. Considering the user convenience perspective. Reducing the number of time classes generally comes with a reduction of the users ride time. Nevertheless, we note that it was necessary to increase the average ride time to find a solution with one time class.

\begin{tabular}{cccccc}
\hline Inconsistency & Cost & $\begin{array}{c}\text { Avg route } \\
\text { duration (hour) }\end{array}$ & $\begin{array}{c}\text { Avg ride } \\
\text { time (min) }\end{array}$ & $\begin{array}{c}\text { Nb. routes } \\
\text { per day }\end{array}$ & $\begin{array}{c}\text { Total Nb. } \\
\text { of routes }\end{array}$ \\
\hline$[3,20,37]$ & 1291.03 & 2.08 & 22.97 & {$[4,4,3,5,3]$} & 19 \\
{$[0,22,38]$} & 1291.73 & 1.97 & 22.49 & {$[4,4,3,5,4]$} & 20 \\
{$[0,17,43]$} & 1304.34 & 1.81 & 21.83 & {$[4,4,5,5,4]$} & 22 \\
{$[0,13,47]$} & 1317.20 & 1.82 & 21.30 & {$[4,4,5,5,4]$} & 22 \\
{$[0,2,58]$} & 1321.87 & 1.82 & 21.48 & {$[4,5,4,5,4]$} & 22 \\
{$[0,1,59]$} & 1331.94 & 1.75 & 21.36 & {$[5,5,4,5,4]$} & 23 \\
{$[0,0,60]$} & 1348.87 & 1.78 & 22.00 & {$[5,5,4,5,4]$} & 23 \\
\hline
\end{tabular}

Table 10: Solution's statistics instance TCDARP_01_60

Figure 12 shows how the consistency requirement concretely modifies the service times for some 
users. This figure represents the times at which two users are picked-up in several solutions of instance TCDARP_01_60. The x-axis represents the time line. On each horizontal line, the five weekly service times of users $\overline{1}$ and 2 are represented. Each line represents different non-dominated solutions denoted $s_{1}, \ldots, s_{5}$, in which the users have one, two or three classes.

In solution $s_{1}$, user 1 has 3 classes with service times varying from 8:00 to 8:30. One time class (class 3 - around 8:27) gathers 3 services out of 5 . In solution $s_{2}$, with 2 time classes, 4 service times out of 5 belong to time class 2 , centered around 8:28. In solution $s_{3}$, all service times are centered around 8:28. A similar phenomenon is observed for user 2. To move from solution $s_{4}$ with two time classes to solution $s_{5}$ with one time class, the algorithm finds routes to serve the user within the time class with the greatest number of service times in $s_{4}$.

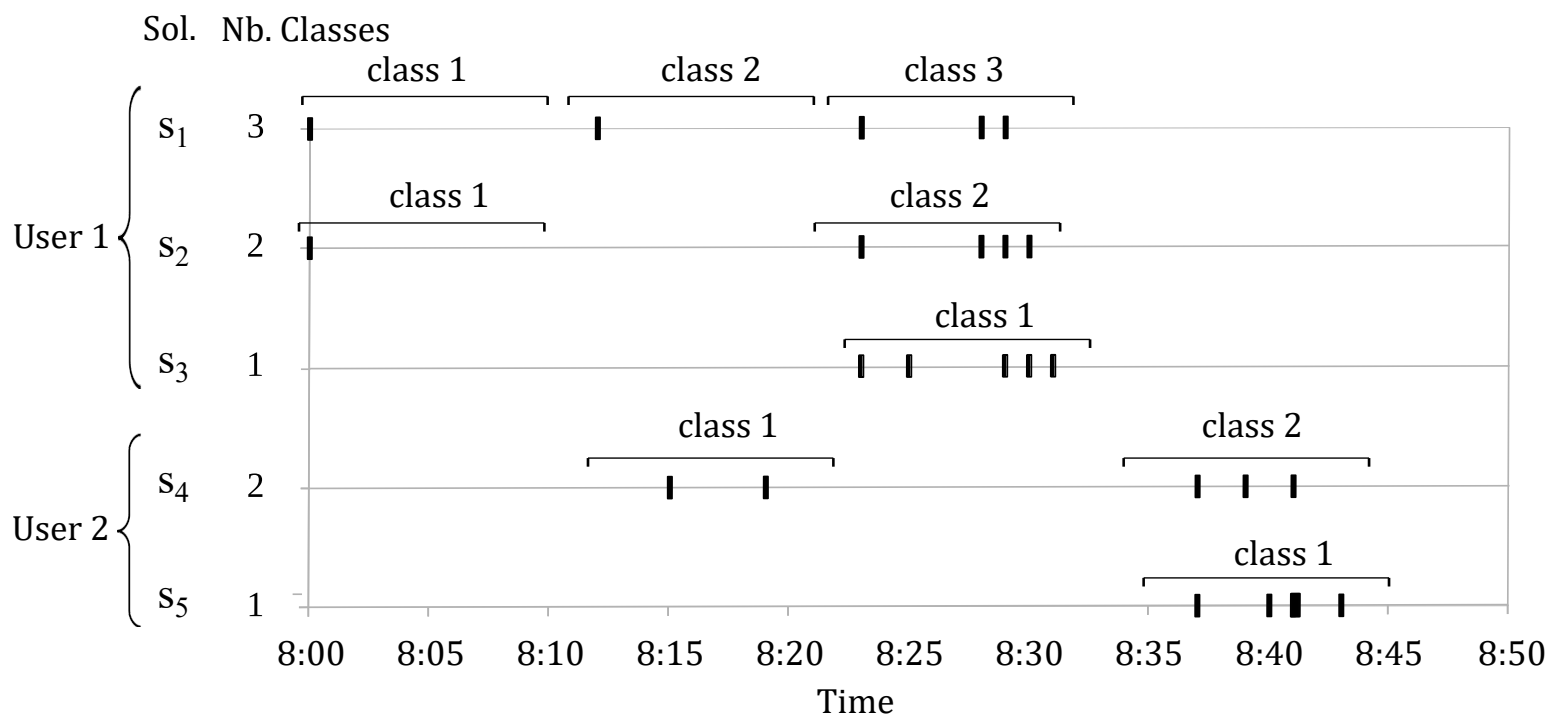

Figure 12: Schedule improvement for 2 users of instance TCDARP_01_60

\subsection{Economic impact of shifting route departure times}

In this section we compute the impact of having flexible route departure times on cost and time consistency. This effect has been studied by Kovacs et al. [14 for the conVRP, showing that departure time flexibility provides considerable improvement in the solution quality under tight consistency requirements. As far as the TC-DARP is concerned, the departure flexibility is limited by time windows and maximum ride-time constraints. The departure of a route can be scheduled at any time between its earliest and its latest departure date. We define the maximum time shift of a route as the difference between these two schedules. The maximum time shift of a route $\omega$ is denoted by $\Delta_{\omega}^{+}$.

Table 11 measures the impact of the departure time shift on the complete set of Synergihp RhôneAlpes instances. Columns 2-4 present the best results on 5 runs of the SPEC when the route time shift is allowed, for values of $C_{\max }$ decreasing from 3 to 1 . Missing values in column 2 mean that all solutions found with $C_{\max }=3$ were dominated by another solution with $C_{\max }=2$. Column 5 (\%R-Shift) shows the percentage of routes in which a departure time shift is actually implemented. Columns 6-8 present the minimum gap on 5 runs with respect to the minimum cost found by the $\mathrm{SP} \varepsilon \mathrm{C}$ when no time shift is allowed $\left(\Delta_{\omega}^{+}=0\right)$. The last row shows the average values.

Since the solution method is heuristic, some some small negative gaps can be observed for $\Delta_{\omega}^{+}=0$. If the solutions found were optimal, negative gaps would not exist. These results show that shifting time departure of routes yields $2.79 \%$ savings on average, with values ranging from $0 \%$ to $9 \%$. According to these results, time consistency can be achieved at a lower cost when route departure times are not fixed in advance. This also implies that shifting time departure can be a lever to improve time consistency without significantly increasing transportation costs in a DARP context. 


\begin{tabular}{|c|c|c|c|c|c|c|c|}
\hline \multirow[t]{2}{*}{ Instance } & \multicolumn{4}{|c|}{$\mathrm{SP} \varepsilon \mathrm{C}$} & \multicolumn{3}{|c|}{$\operatorname{SP} \varepsilon \mathrm{C}\left(\Delta_{\omega}^{+}=0\right)$} \\
\hline & 3 & 2 & 1 & \%R-Shift & 3 & 2 & 1 \\
\hline TCDARP_00_80 & 2045.54 & 2044.43 & 2061.89 & $49 \%$ & $-0.07 \%$ & $0.25 \%$ & $0.35 \%$ \\
\hline TCDARP_01_60 & 1291.03 & 1291.73 & 1348.87 & $40 \%$ & $0.18 \%$ & $0.48 \%$ & $0.20 \%$ \\
\hline TCDARP_02_80 & 2523.21 & 2533.86 & 2561.40 & $31 \%$ & $0.00 \%$ & $-0.01 \%$ & $0.31 \%$ \\
\hline TCDARP_03_70 & & 1735.07 & 1752.43 & $33 \%$ & & $0.00 \%$ & $0.30 \%$ \\
\hline TCDARP_04_80 & & 1207.72 & 1220.91 & $32 \%$ & & $0.00 \%$ & $2.44 \%$ \\
\hline TCDARP_05_80 & 1871.25 & 1871.53 & 1923.21 & $40 \%$ & $0.37 \%$ & $0.06 \%$ & $0.08 \%$ \\
\hline TCDARP_06_60 & & 3304.41 & 3332.21 & $42 \%$ & & $0.00 \%$ & $0.54 \%$ \\
\hline TCDARP_07_65 & 1865.46 & 1868.15 & 1920.95 & $50 \%$ & $0.00 \%$ & $-0.07 \%$ & $3.22 \%$ \\
\hline TCDARP_08_120 & & 5500.45 & 5534.44 & $39 \%$ & & $0.02 \%$ & $1.76 \%$ \\
\hline TCDARP_09_135 & 2857.18 & 2880.57 & 3107.12 & $38 \%$ & $0.27 \%$ & $-0.18 \%$ & $1.17 \%$ \\
\hline TCDARP_10_160 & & 2621.35 & 2779.06 & $42 \%$ & $0.03 \%$ & $0.00 \%$ & $8.68 \%$ \\
\hline TCDARP_11_160 & 3222.10 & 3233.17 & 3549.53 & $42 \%$ & $0.21 \%$ & $0.00 \%$ & $4.84 \%$ \\
\hline TCDARP_12_280 & & 7724.32 & 8533.52 & $42 \%$ & & $0.27 \%$ & $8.93 \%$ \\
\hline TCDARP_13_295 & 6314.24 & 6329.57 & 7465.76 & $47 \%$ & $-0.08 \%$ & $0.08 \%$ & $6.16 \%$ \\
\hline & & & Avg & $41 \%$ & $0.10 \%$ & $0.07 \%$ & $2.79 \%$ \\
\hline
\end{tabular}

Table 11: Economic implications of allowing a later departure of routes (Best solutions on 5 runs)

\section{Conclusions}

This paper introduces a new variant of the DARP denoted the time consistent DARP. It aims to find trade-off solutions between two objectives: the transportation cost and the time consistency of users. Regarding the literature on the topic, we propose a new formulation of the time consistency of a solution: for a particular user, the number of time classes expresses the number of significantly different service times within one week. We calculate the time consistency of a solution as a lexicographic function of the number of time classes per user. Regarding optimization methods, this formulation is more time consuming than a traditional min-max objective, but it returns a more detailed Pareto front approximation that helps windows and maximum ride times in a problem with multiple destinations. This problem was studied in the context of door-to-door transportation of children with disabilities in region Auvergne-Rhône-Alpes in France.

To compute Pareto front approximations, we developed a matheuristic framework called SPEC based on an epsilon constraint procedure and a set partitioning problem. An initial set of routes is produced by a LNS matheuristic previously proposed for the FSM-DARP-RC in Tellez et al. 37. Additional routes are generated by an extension of this algorithm to the DARP with multiple time windows and minimal waiting time. At each iteration, a subset of routes is chosen to feed the $\mathrm{SP} \varepsilon \mathrm{C}$ procedure. Experiments show the high performance of the $\mathrm{SP} \varepsilon \mathrm{C}$ on real-life instances for up to 295 users. $\mathrm{SP} \varepsilon \mathrm{C}$ was also been tested on literature instances and was shown to improve the state-of-the-art algorithm for the TC-VRP benchmark.

Some users with disabilities are very sensitive to inconsistent schedules. In this study, we show that economic solutions are already fairly consistent with very few users having 3 time classes. In addition, we found that in most instances, with a small increase in transportation costs $(<1 \%)$, users schedules with at most 2 time classes are possible. Finally, we show that allowing a flexible departure of routes improves the transportation costs of highly consistent solutions. Future researches concern implementing additional features of the real-life problem such as driver-related constraints (working time, breaks, regulation and driver-consistency) and heterogeneous fleet.

\section{Acknowledgements}

750 We would like to thank the European Union through the European Regional Development Fund (ERDF) and the French region Auvergne-Rhône-Alpes for their financial support of the NOMAd project. 


\section{A Appendix}

\section{A.1 Generation of multiple time windows: $\operatorname{gen} \operatorname{MTW}(\mathcal{H}, u)$}

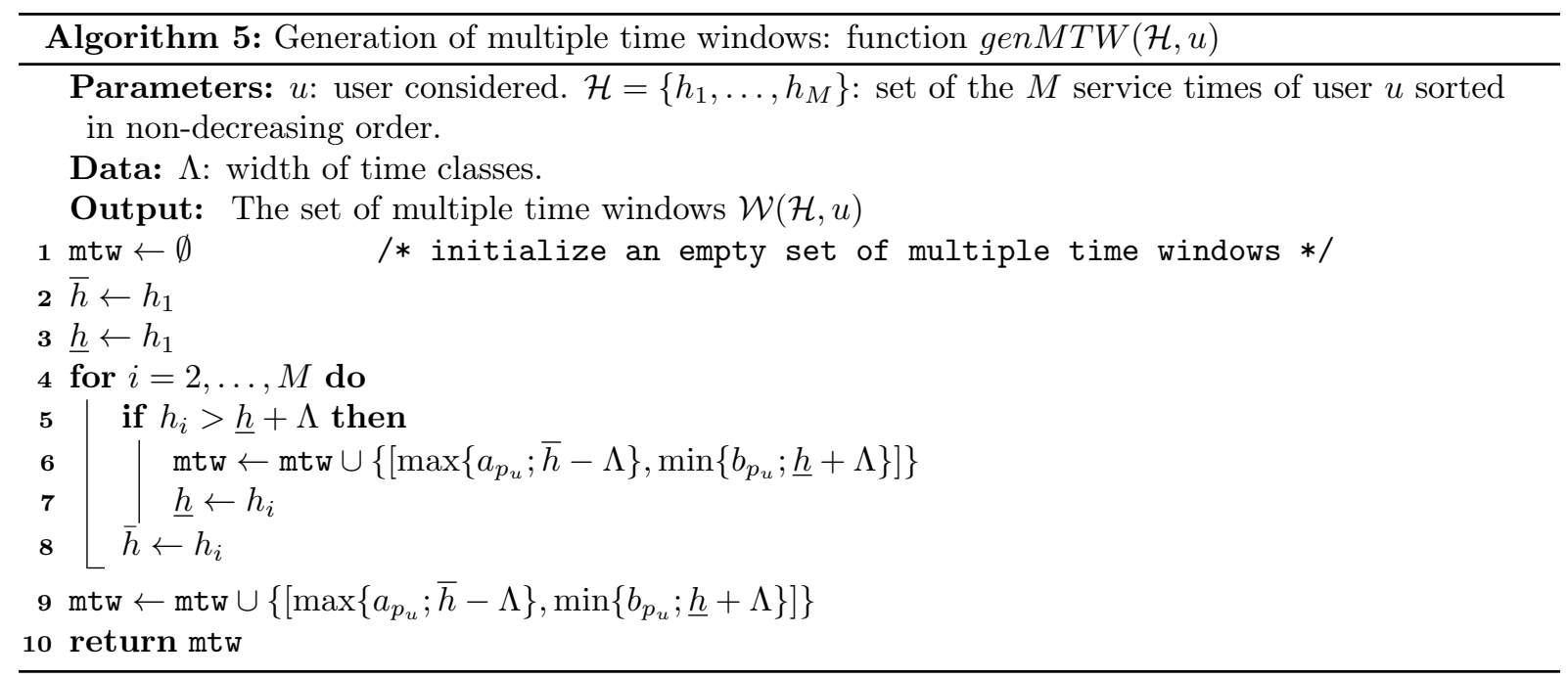




\section{A.2 Scheduling algorithm}

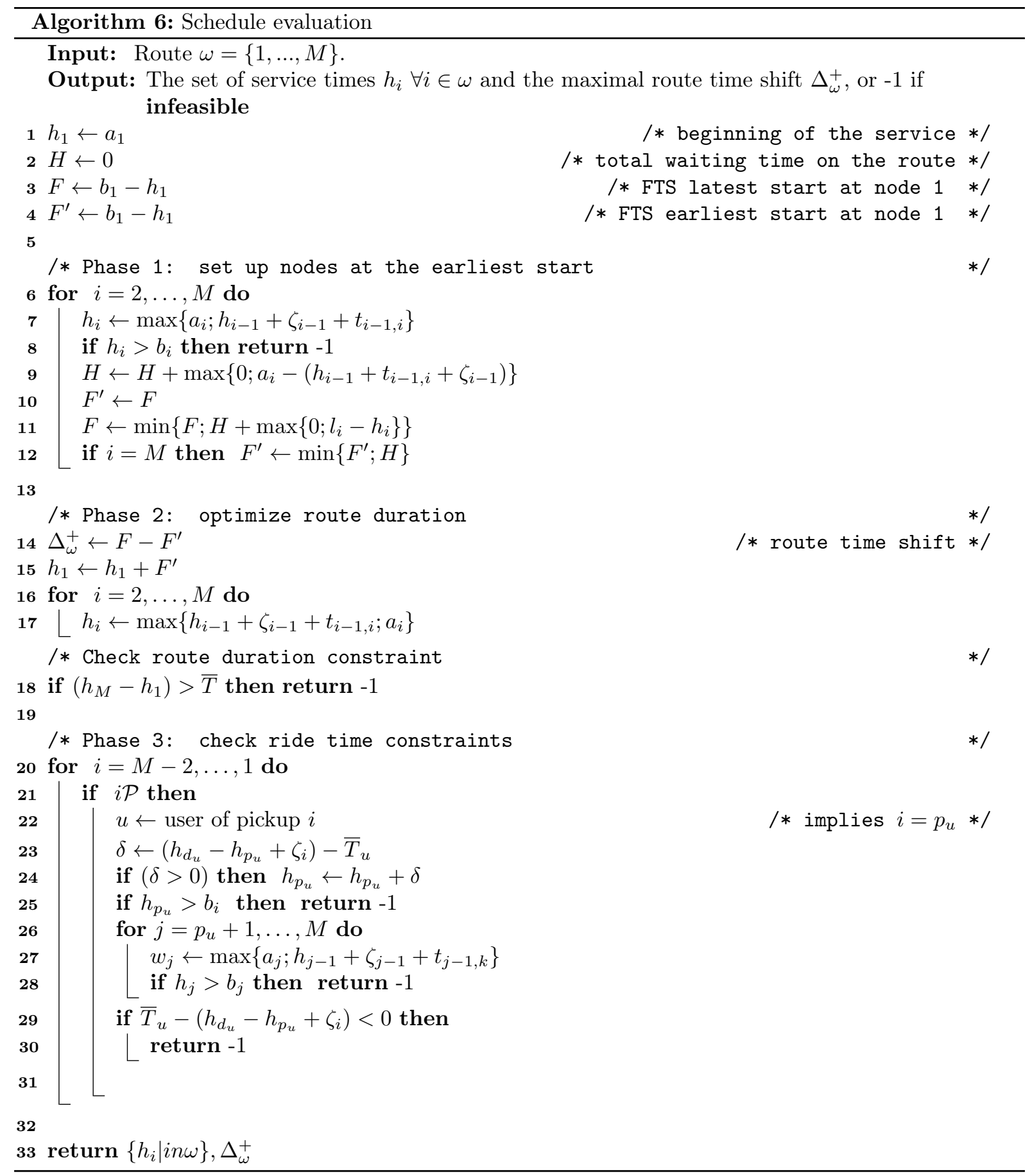


${ }_{755}$ A.3 Parameters LSN-SCP

\begin{tabular}{l|l}
\hline Parameters to generate pool $\mathcal{L}$ \\
\hline$\chi=5 \%$ & record-to-record acceptance criterion. \\
penalty $=10000$ & penalty cost for incomplete solutions. \\
$\Phi^{-}=10 \%$ & minimal proportion of removed request used by removal operators. \\
$\Phi^{+}=45 \%$ & maximal proportion of removed request used by removal operators. \\
$p=6$ & roulette wheel parameter for the historical node-pair operator. \\
$\sigma_{\text {init }}^{+}=4$-regret & repair operator for building the initial solution \\
$\eta=1000$ & launch frequency of the SCP. \\
Iters $=10000$ & max number of iterations. \\
$\psi=1.25$ & RSCP coefficient to recompute the launch frequency of the SCP. \\
\hline New parameters to generate pool $\mathcal{L}_{\text {new }}$ \\
\hline Iters $=250$ & max number of iterations. \\
$\eta=\infty$ & the SCP is deactivated. \\
\hline
\end{tabular}

Table 12: Parameters LNS-SCP 37. 


\section{B TC-VRP}

\begin{tabular}{|c|c|c|c|c|c|c|}
\hline \multirow{2}{*}{$\begin{array}{l}\text { Instance } \\
\qquad C_{\max }\end{array}$} & \multicolumn{5}{|c|}{ Transportation cost } & \multirow{2}{*}{$\begin{array}{l}\text { Time } \\
(\min )\end{array}$} \\
\hline & $\leq 5$ & $\leq 4$ & $\leq 3$ & $\leq 2$ & $\leq 1$ & \\
\hline data $5-15$ & 663.2 & 663.2 & 663.2 & 674.1 & 782.3 & 11.6 \\
\hline data $5-21$ & 773.7 & 779.1 & 779.1 & 779.1 & 817.3 & 21.9 \\
\hline data5-25 & 617.4 & 617.4 & 617.4 & 622.2 & 669.0 & 29.1 \\
\hline data5-26 & 767.6 & 767.6 & 771.9 & 778.8 & 815.9 & 45.8 \\
\hline data $5-27$ & 934.6 & 934.6 & 934.6 & 942.0 & 1026.1 & 61.2 \\
\hline data5-32 & 984.9 & 984.9 & 984.9 & 989.9 & 1034.5 & 22.1 \\
\hline data5-41 & 1420.7 & 1420.7 & 1422.6 & 1461.6 & 1615.9 & 126.9 \\
\hline data $5-44$ & 1142.9 & 1142.9 & 1142.9 & 1149.4 & 1220.3 & 74.3 \\
\hline data5-46 & 1458.9 & 1458.9 & 1465.4 & 1492.9 & 1607.2 & 114.4 \\
\hline data5-48 & 1440.8 & 1449.4 & 1452.4 & 1459.8 & 1597.8 & 154.9 \\
\hline data $5-55$ & 1569.1 & 1569.1 & 1571.1 & 1581.0 & 1696.9 & 139.4 \\
\hline data5-59 & 2714.8 & 2721.5 & 2721.5 & 2883.6 & 3115.9 & 281.3 \\
\hline data5-64 & 2082.0 & 2082.0 & 2100.8 & 2112.4 & 2304.2 & 118.9 \\
\hline data5-65 & 1759.4 & 1759.4 & 1766.2 & 1777.1 & 1923.9 & 128.6 \\
\hline data6-15 & 689.4 & 689.4 & 689.4 & 695.6 & 741.3 & 2.2 \\
\hline data6-21 & 792.0 & 792.0 & 796.2 & 798.1 & 831.7 & 40.9 \\
\hline data6-25 & 680.9 & 680.9 & 683.5 & 683.5 & 728.7 & 32.9 \\
\hline data6-26 & 838.7 & 838.7 & 838.7 & 843.2 & 905.0 & 56.0 \\
\hline data6-27 & 949.8 & 949.8 & 949.8 & 954.0 & 1060.3 & 57.9 \\
\hline data6-32 & 991.1 & 991.1 & 991.1 & 996.2 & 1013.5 & 10.4 \\
\hline data6-41 & 1500.3 & 1500.3 & 1504.2 & 1506.7 & 1735.5 & 92.8 \\
\hline data6-44 & 1239.1 & 1239.1 & 1243.9 & 1244.6 & 1405.9 & 76.7 \\
\hline data6-46 & 1485.1 & 1485.1 & 1496.6 & 1526.0 & 1597.0 & 93.0 \\
\hline data6-48 & 1507.4 & 1507.4 & 1519.4 & 1531.6 & 1702.8 & 158.5 \\
\hline data $6-55$ & 1816.8 & 1816.8 & 1826.3 & 1854.4 & 1987.7 & 121.0 \\
\hline data6-59 & 2931.3 & 2931.3 & 2933.0 & 3037.4 & 3389.6 & 204.1 \\
\hline data6-64 & 2264.3 & 2264.3 & 2271.0 & 2305.6 & 2527.0 & 100.6 \\
\hline data6-65 & 1981.9 & 1981.9 & 1990.6 & 1998.3 & 2219.5 & 163.6 \\
\hline
\end{tabular}

Table 13: Benchmark of 9 


\begin{tabular}{|c|c|c|c|c|c|c|}
\hline \multirow{2}{*}{$\begin{array}{l}\text { Instance } \\
C_{\max }\end{array}$} & \multicolumn{5}{|c|}{ Transportation cost } & \multirow{2}{*}{$\begin{array}{l}\text { Time } \\
(\min ) \\
\end{array}$} \\
\hline & $\leq 5$ & $\leq 4$ & $\leq 3$ & $\leq 2$ & $\leq 1$ & \\
\hline data7-15 & 746.9 & 746.9 & 746.9 & 752.6 & 790.3 & 11.9 \\
\hline data7-21 & 830.1 & 833.7 & 833.7 & 833.7 & 899.3 & 39.8 \\
\hline data7-25 & 719.5 & 724.7 & 724.7 & 728.8 & 767.9 & 34.9 \\
\hline data7-26 & 880.3 & 880.3 & 883.5 & 887.4 & 920.7 & 37.9 \\
\hline data7-27 & 1053.4 & 1053.4 & 1053.4 & 1056.5 & 1108.5 & 39.7 \\
\hline data7-32 & 1079.7 & 1079.7 & 1079.7 & 1097.3 & 1154.3 & 57.2 \\
\hline data7-41 & 1644.8 & 1644.8 & 1648.5 & 1661.0 & 1730.4 & 63.2 \\
\hline data7-44 & 1295.9 & 1295.9 & 1300.3 & 1307.0 & 1311.7 & 20.5 \\
\hline data7-46 & 1648.6 & 1648.6 & 1650.1 & 1664.6 & 1715.9 & 72.4 \\
\hline data7-48 & 1687.1 & 1698.9 & 1698.9 & 1712.0 & 1774.3 & 116.6 \\
\hline data7-55 & 1889.0 & 1889.0 & 1896.3 & 1918.0 & 2036.0 & 136.5 \\
\hline data7-59 & 3262.4 & 3262.4 & 3307.4 & 3596.6 & 3892.4 & 725.2 \\
\hline data7-64 & 2552.9 & 2552.9 & 2552.9 & 2583.6 & 2823.4 & 136.9 \\
\hline data7-65 & 2196.0 & 2196.0 & 2196.0 & 2224.2 & 2347.3 & 159.8 \\
\hline data8-15 & 773.9 & 773.9 & 773.9 & 780.2 & 808.5 & 10.8 \\
\hline data8-21 & 898.4 & 898.4 & 898.4 & 905.0 & 956.3 & 37.8 \\
\hline data8-25 & 853.3 & 853.3 & 853.3 & 853.3 & 857.0 & 0.2 \\
\hline data8-26 & 962.5 & 962.5 & 962.5 & 970.8 & 998.2 & 35.2 \\
\hline data8-27 & 1184.8 & 1193.9 & 1193.9 & 1193.9 & 1220.6 & 17.6 \\
\hline data8-32 & 1144.0 & 1144.0 & 1152.8 & 1152.8 & 1181.7 & 35.8 \\
\hline data8-41 & 1886.8 & 1886.8 & 1888.5 & 1898.0 & 1954.3 & 63.9 \\
\hline data8-44 & 1409.0 & 1409.0 & 1409.0 & 1414.9 & 1441.2 & 28.8 \\
\hline data8-46 & 1758.5 & 1758.5 & 1772.9 & 1774.8 & 1817.2 & 33.8 \\
\hline data8-48 & 1815.7 & 1815.7 & 1820.9 & 1824.4 & 1898.1 & 125.7 \\
\hline data8 -55 & 2007.7 & 2007.7 & 2015.4 & 2037.2 & 2104.0 & 120.0 \\
\hline data8-59 & 3545.7 & 3545.7 & 3580.5 & 3874.4 & 4020.5 & 427.4 \\
\hline data8-64 & 2723.3 & 2723.3 & 2723.3 & 2743.0 & 2978.2 & 126.8 \\
\hline data8-65 & 2404.2 & 2404.2 & 2422.9 & 2433.5 & 2524.4 & 159.5 \\
\hline data9-15 & 797.5 & 797.5 & 797.5 & 804.3 & 816.1 & 3.6 \\
\hline data9-21 & 998.6 & 998.6 & 998.6 & 1003.0 & 1008.9 & 5.7 \\
\hline data9-25 & 894.6 & 894.6 & 894.6 & 894.6 & 908.1 & 1.6 \\
\hline data9-26 & 1024.6 & 1024.6 & 1024.6 & 1024.6 & 1028.0 & 0.4 \\
\hline data9-27 & 1210.6 & 1210.6 & 1210.6 & 1219.5 & 1241.9 & 12.3 \\
\hline data9-32 & 1187.7 & 1187.7 & 1199.4 & 1199.4 & 1204.9 & 6.4 \\
\hline data9-41 & 2022.8 & 2022.8 & 2022.8 & 2022.8 & 2032.1 & 6.3 \\
\hline data9-44 & 1532.5 & 1532.5 & 1532.5 & 1532.5 & 1595.1 & 54.1 \\
\hline data9-46 & 1827.0 & 1827.0 & 1827.0 & 1841.6 & 1871.0 & 30.7 \\
\hline data9-48 & 1973.5 & 1973.5 & 1973.5 & 1992.8 & 2007.7 & 28.6 \\
\hline data9-55 & 2176.0 & 2176.0 & 2176.0 & 2188.1 & 2275.9 & 70.3 \\
\hline data9-59 & 3913.0 & 3913.0 & 3916.3 & 3946.6 & 4025.3 & 96.3 \\
\hline data9-64 & 2942.6 & 2942.6 & 2964.9 & 2964.9 & 2999.6 & 28.8 \\
\hline data9-65 & 2586.5 & 2586.5 & 2586.5 & 2604.7 & 2624.2 & 68.0 \\
\hline
\end{tabular}

Table 14: Benchmark of 9 


\section{References}

[1] ANAP. Améliorer la gestion des transports de personnes handicapées. Français. Tech. rep. Appui santé \& médico social, 2016.

[2] Kris Braekers and Attila A. Kovacs. "A multi-period dial-a-ride problem with driver consistency". In: Transportation Research Part B: Methodological 94 (Dec. 2016), pp. 355-377.

[3] Stefan Bunte and Natalia Kliewer. "An overview on vehicle scheduling models". In: Public Transport 1.4 (Nov. 2009), pp. 299-317.

[4] Samuela Carosi et al. "A matheuristic for integrated timetabling and vehicle scheduling". In: Transportation Research Part B: Methodological 127 (Sept. 2019), pp. 99-124.

[5] Vira Chankong and Yacov Y Haimes. Multiobjective decision making: theory and methodology. Courier Dover Publications, 2008.

[6] Karl F. Doerner and Juan-José Salazar-González. "Chapter 7: Pickup-and-Delivery Problems for People Transportation". In: Vehicle Routing. Ed. by Paolo Toth and Daniele Vigo. Philadelphia, PA: Society for Industrial and Applied Mathematics, Nov. 2014, pp. 193-212.

[7] Michael Drexl. "Synchronization in Vehicle Routing-A Survey of VRPs with Multiple Synchronization Constraints". In: Transportation Science 46.3 (Aug. 2012), pp. 297-316.

[8] Alan L. Erera, Martin Savelsbergh, and Emrah Uyar. "Fixed routes with backup vehicles for stochastic vehicle routing problems with time constraints". In: Networks 54.4 (Dec. 2009), pp. 270-283.

[9] Dominique Feillet et al. "A new consistent vehicle routing problem for the transportation of people with disabilities". In: Networks 63.3 (May 2014), pp. 211-224.

[10] Philippe Grangier et al. "A matheuristic based on large neighborhood search for the vehicle routing problem with cross-docking". In: Computers $\& 3$ Operations Research (2017).

[11] Chris Groër, Bruce Golden, and Edward Wasil. "The Consistent Vehicle Routing Problem". In: Manufacturing \&3 Service Operations Management 11.4 (Oct. 2009), pp. 630-643.

[12] Y. Haimes, L. Lasdon, and D. Wismer. "On a bicriterion formulation of the problems of integrated system identification and system optimization". In: IEEE Transactions on Systems, Man, and Cybernetics 1 (1971), pp. 296-297.

[13] Sin C. Ho et al. "A survey of dial-a-ride problems: Literature review and recent developments". In: Transportation Research Part B: Methodological 111 (2018), pp. 395-421.

[14] Attila A. Kovacs, Sophie N. Parragh, and Richard F. Hartl. "A template-based adaptive large neighborhood search for the consistent vehicle routing problem". In: Networks 63.1 (Jan. 2014), pp. $60-81$.

[15] Attila A. Kovacs et al. "The Generalized Consistent Vehicle Routing Problem". In: Transportation Science 49.4 (Nov. 2015), pp. 796-816.

[16] Attila A. Kovacs et al. "Vehicle routing problems in which consistency considerations are important: A survey". In: Networks 64.3 (Oct. 2014), pp. 192-213.

[17] Fabien Lehuédé et al. "A multi-criteria large neighbourhood search for the transportation of disabled people". In: Journal of the Operational Research Society 65.7 (2014), pp. 983-1000.

[18] Hongtao Lei, Gilbert Laporte, and Bo Guo. "Districting for routing with stochastic customers". In: EURO Journal on Transportation and Logistics 1.1-2 (2012), pp. 67-85.

[19] Zhixing Luo et al. "On service consistency in multi-period vehicle routing". In: European Journal of Operational Research 243.3 (June 2015), pp. 731-744.

[20] Dennis Luxen and Christian Vetter. "Real-time routing with OpenStreetMap data". In: Proceedings of the 19th ACM SIGSPATIAL International Conference on Advances in Geographic Information Systems. GIS'11. Chicago, Illinois: ACM, 2011, pp. 513-516.

[21] Mathias Michaelis and Anita Schöbel. "Integrating line planning, timetabling, and vehicle scheduling: a customer-oriented heuristic". In: Public Transport 1.3 (Aug. 2009), p. 211.

[22] Ashlea Bennett Milburn and Jessica Spicer. "Multi-objective home health nurse routing with remote monitoring devices". In: International Journal of Planning and Scheduling 1.4 (2013), pp. 242-263.

[23] Yves Molenbruch, Kris Braekers, and An Caris. "Typology and literature review for dial-a-ride problems". In: Annals of Operations Research 259.1-2 (Dec. 2017), pp. 295-325. 
[24] Wlodzimierz Ogryczak et al. "Fair optimization and networks: A survey". In: Journal of Applied Mathematics 2014 (2014).

[25] Junhyuk Park and Byung-In Kim. "The school bus routing problem: A review". In: European Journal of Operational Research 202.2 (Apr. 2010), pp. 311-319.

[26] David Pisinger and Stefan Ropke. "A general heuristic for vehicle routing problems". en. In: Computers \&3 Operations Research 34.8 (2007), pp. 2403-2435. ISSN: 03050548.

[27] Ulrike Ritzinger, Jakob Puchinger, and Richard F Hartl. "A survey on dynamic and stochastic vehicle routing problems". In: International Journal of Production Research 54.1 (2016), pp. 215231.

[28] Verena Schmid and Jan Fabian Ehmke. "Integrated timetabling and vehicle scheduling with balanced departure times". In: OR Spectrum 37.4 (Oct. 2015), pp. 903-928.

[29] Michael Schneider et al. "Territory-Based Vehicle Routing in the Presence of Time-Window Constraints". In: Transportation Science 49.4 (Nov. 2015), pp. 732-751.

[30] Karen Smilowitz, Maciek Nowak, and Tingting Jiang. "Workforce Management in Periodic Delivery Operations". In: Transportation Science 47.2 (May 2013), pp. 214-230.

[31] Remy Spliet and Guy Desaulniers. "The discrete time window assignment vehicle routing problem". In: European Journal of Operational Research 244.2 (2015), pp. 379-391.

${ }_{825}[32]$ Remy Spliet and Adriana F. Gabor. "The Time Window Assignment Vehicle Routing Problem". en. In: Transportation Science 49.4 (Nov. 2015), pp. 721-731.

[33] Anirudh Subramanyam and Chrysanthos E Gounaris. "A branch-and-cut framework for the consistent traveling salesman problem". In: European Journal of Operational Research 248.2 (2016), pp. 384-395.

[34] Ilgaz Sungur et al. "A Model and Algorithm for the Courier Delivery Problem with Uncertainty". In: Transportation Science 44.2 (May 2010), pp. 193-205.

[35] C.D. Tarantilis, F. Stavropoulou, and P.P. Repoussis. "A template-based Tabu Search algorithm for the Consistent Vehicle Routing Problem". In: Expert Systems with Applications 39.4 (Mar. 2012), pp. $4233-4239$.

[36] Oscar A. Téllez Sánchez. "Optimisation du transport quotidien des personnes en situation de handicap". PhD thesis. INSA Lyon, Sept. 2019.

[37] Oscar Tellez et al. "The fleet size and mix dial-a-ride problem with reconfigurable vehicle capacity". In: Transportation Research Part C: Emerging Technologies 91 (2018), pp. 99-123.

[38] Paolo Toth and Daniele Vigo. "Heuristic algorithms for the handicapped persons transportation problem". In: Transportation Science 31.1 (1997), pp. 60-71.

[39] Zefeng Xu and Yanguang Cai. "Variable neighborhood search for consistent vehicle routing problem". In: Expert Systems with Applications 113 (Dec. 2018), pp. 66-76. ISSN: 09574174.

[40] Hongsheng Zhong, Randolph W. Hall, and Maged Dessouky. "Territory Planning and Vehicle Dispatching with Driver Learning". In: Transportation Science 41.1 (Feb. 2007), pp. 74-89. 